\title{
Coherent structures in wall-bounded turbulence
}

\author{
DAVID J.C. DENNIS \\ School of Engineering, University of Liverpool, L69 3GH, Liverpool, United Kingdom \\ Manuscript received on November 18, 2014; accepted for publication on February 12, 2015
}

\begin{abstract}
The inherent difficulty of understanding turbulence has led to researchers attacking the topic in many different ways over the years of turbulence research. Some approaches have been more successful than others, but most only deal with part of the problem. One approach that has seen reasonable success (or at least popularity) is that of attempting to deconstruct the complex and disorganised turbulent flow field into to a set of motions that are in some way organised. These motions are generally called "coherent structures". There are several strands to this approach, from identifying the coherent structures within the flow, defining their characteristics, explaining how they are created, sustained and destroyed, to utilising their features to model the turbulent flow. This review considers research on coherent structures in wallbounded turbulent flows: a class of flow which is extremely interesting to many scientists (mainly, but not exclusively, physicists and engineers) due to their prevalence in nature, industry and everyday life. This area has seen a lot of activity, particularly in recent years, much of which has been driven by advances in experimental and computational techniques. However, several ideas, developed many years ago based on flow visualisation and intuition, are still both informative and relevant. Indeed, much of the more recent research is firmly indebted to some of the early pioneers of the coherent structures approach. Therefore, in this review, selected historical research is discussed along with the more contemporary advances in an attempt to provide the reader with a good overview of how the field has developed and to highlight the perspicacity of some of the early researchers, as well as providing an overview of our current understanding of the role of coherent structures in wall-bounded turbulent flows.
\end{abstract}

Key words: turbulence, wall bounded flows, coherent structures, particle image velocimetry.

\section{INTRODUCTION}

Wall-bounded turbulence is physically present in many types of flow, research conducted in the canonical flows are under consideration in this review, namely: steady, fully developed, incompressible, smooth-wall pipe and channel flow, and zero pressure gradient boundary layer. The effects of roughness, riblets, flow control

Correspondence to: David J.C. Dennis

E-mail: david.dennis@liverpool.ac.uk devices, particulates, additives or pressure gradients are not considered. For many years research into wall-bounded turbulence was focussed on the measurement and interpretation of average quantities, such as the mean velocity profile, the mean wall shear stress and other statistical quantities. This review does not consider these aspects, but focusses on attempts to capture coherent structures in wall-bounded flow and the models and viewpoints that these structures have inspired. 
The initiation of this line of research can be traced back to Kline et al. (1967) and Townsend (1976). Researchers in these early days were restricted by the experimental measurement techniques available to them and in recent years much progress has been made in this area using modern techniques. This review considers selected historical work as well as more contemporary research. Hopefully this will provide a reader with an appreciation for how the field has developed as well as revealing how insightful some of the early researchers were, despite the technological limitations they faced.

A variety of coherent motions have been observed in wall-bounded turbulent flow and these observations have led to an area of research that attempts to break down complex turbulent flow into organised, elementary motions. The very early turbulence pioneers did not consider coherent motions in their studies of turbulence, as pointed out in Cantwell (1981), "Although the concept of an eddy played a central role in constructing mathematical models of turbulence, it was essentially an abstraction and early work on turbulence is marked by a singular lack of any attempt to sketch or schematically visualize turbulent motion." The interest in coherent motions was largely sparked by flow visualisation that enabled structural features of turbulent flow to be observed.

Before delving into the topic of coherent structures it would be ideal to give an unambiguous and universally accepted definition of a coherent structure. Unfortunately no such definition exists. Indeed, the concept of a coherent structure is essentially subjective and nomenclature has been a thorny issue for many years. Although any definition given here would be somewhat personal and heuristic, it seems remiss not to attempt to give a definition of a coherent structure which at least applies to this review. In order to do this we can first examine the two words individually. "Coherent" implies both consistency and something that forms part of a unified whole. "Structure" indicates something that is composed of parts arranged in some organised way.
Thus, the main characteristic of coherent structures is their consistency (for example a consistent pattern in the velocity field) and their main influence is to construct the turbulent flow through some degree of organisation. Using this viewpoint we could define coherent structures as the building-blocks of turbulent flow: and we have two main questions to answer. What are the characteristics of these building-blocks and how are they organised? All the research discussed in this review is essentially trying to answer these two questions.

The use of ambiguous symbols has been minimised in an attempt to avoid confusion, but inevitably some are required. In general the streamwise, spanwise and wall-normal coordinates are denoted by $x, y$, and $z$ respectively with corresponding velocity fluctuations $u, v$ and $w$. Uppercase $U$ is used for streamwise velocity to distinguish it from the fluctuating quantities. The exceptions from this convention are noted explicitly where appropriate.

\section{ELEMENTARY COHERENT STRUCTURES}

In this section experiments that reveal the elementary coherent structures in wall-bounded turbulence are reviewed. The term "elementary" is used to distinguish these structures as fundamental building blocks in the sense that they are not thought to be agglomerations of other structures.

\section{STREAKS, BURSTS, SWEEPS AND EJECTIONS}

Low-speed streaks were one of the earliest discovered features of turbulent flow next to boundaries that could be regarded as coherent structures (Kline et al. 1967). These are elongated, streamwise-oriented regions of low-speed fluid (sometimes extending to over 1000 viscous lengths), ubiquitous in the nearwall region of turbulent flows. In figure 1, streaks in a water flow are highlighted by electrolysis-created micro-air bubbles at $y^{+}=5$ above the wall. Average spacing between streaks is 100 viscous lengths (although the standard deviation is quite high) (Gupta et al. 1971, Smith and Metzler 1983). 


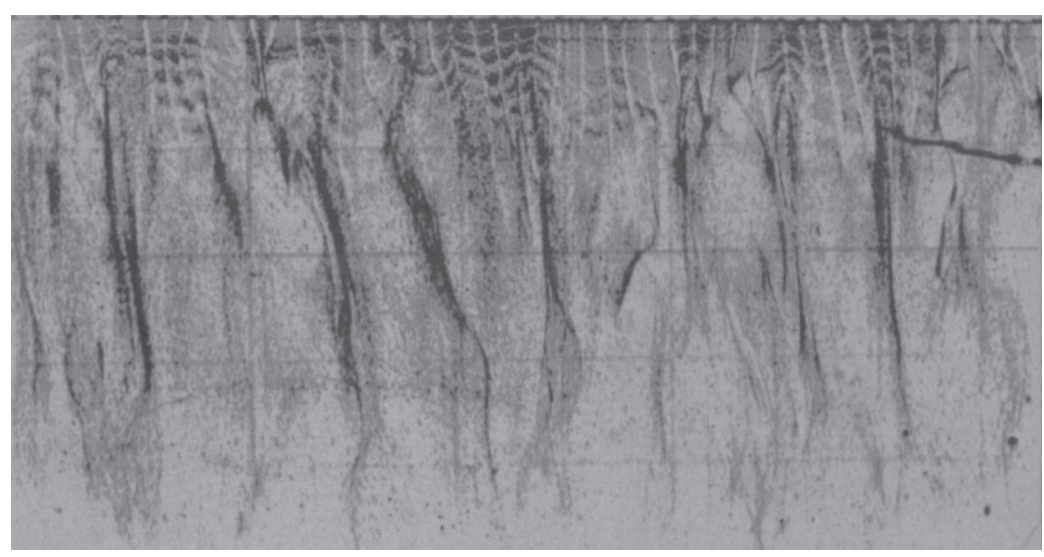

Figure 1 - Low-speed streaks (from Kline et al. 1967). Flow is from top to bottom.

A related feature is that of the turbulent burst (Kline et at. 1967). This is said to be as a result of a streak lifting up away from the wall and oscillating in the buffer layer $\left(8 \leq y^{+} \leq 12\right)$ before breaking up in the region $10 \leq y^{+} \leq 30$. Considering this "ejection" process, it is of lowspeed fluid, thus $u<0$, departing from the vicinity of the boundary, hence $v>0$, which is consistent with the observation of positive Reynolds shear stress $(u v<0)$ in the wall region. Similarly, the opposite event, of high speed fluid $(u>0)$ approaching the wall $(v<0)$, also leads to positive shear stress. These events, often called "sweeps" or "inrushes", have been found to contribute to Reynolds shear stress to a similar degree as ejections in general but their relative importance varies with distance from the wall. Ejections contribute more for higher values of $y^{+}$, whereas sweeps are more significant near the surface $\left(y^{+}<15\right)$, which makes sense in that as distance from the wall increases there is a greater depth from which an ejection can originate (Mathieu and Scott. 2000). Figure 2 from Grass (1971) shows the effect of ejections and sweeps on the instantaneous mean velocity profile and wall-normal fluctuation. (Note that a diferent nomenclature is used in the figure, so: $U_{1} \equiv U, x_{2} \equiv y$, and $u_{2} \equiv v$.) The obvious characteristics of ejections and sweeps are present, but as well as that we see the inflectional nature of the velocity profiles. Such inflectional profiles are prone to instability and as such they support the idea of the abrupt break-up of the ejection, i.e. the turbulent burst.

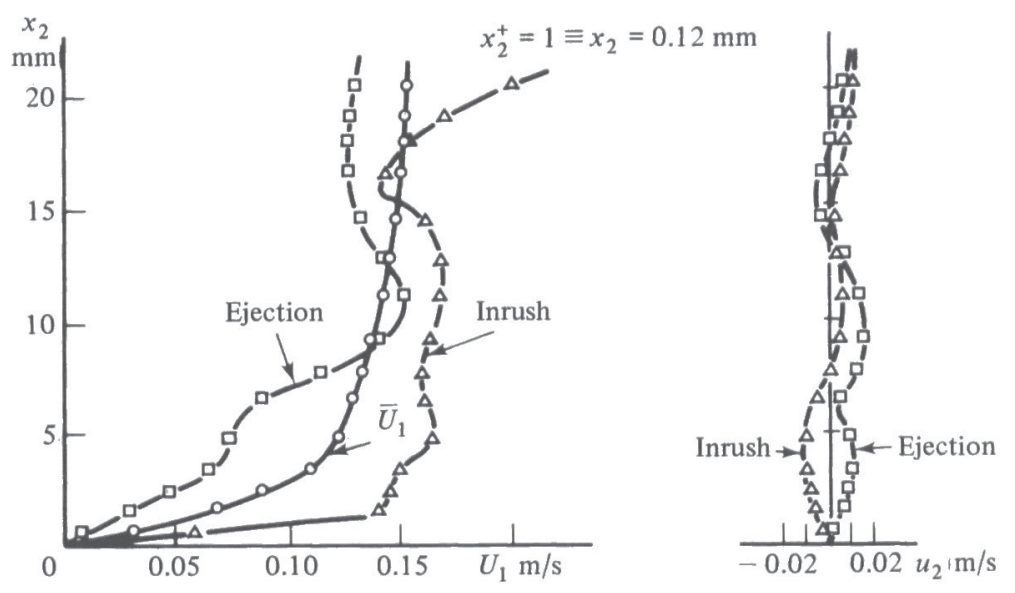

Figure 2 - Instantaneous velocity profiles (from Grass 1971). 
Ejections and sweeps were considered of primary importance in considerations of the generation and maintenance of turbulence. From their visual experiments Corino and Brodkey concluded that, "The most important feature of the wall region is the ejection of fluid elements which occurs there... The interaction of these elements with the mean flow creates turbulence" (Corino and Brodkey. 1969). They are also clearly important because of their contribution to the Reynolds stress, and the relevance of that to the unclosed quantities in the Reynolds-averaged Navier-Stokes equations.

QUASI-STREAMWISE AND SPANWISE VORTICES

Streamwise vortices have traditionally been identified using a single wall-normal-spanwise plane of marked fluid (or of velocity vectors). This method does not distinguish between purely streamwise vortices and those that are tilted, hence the term quasi-streamwise is used. Experiments (for example using hydrogen bubble wires in a low Reynolds number water channel) (Clark and Markland 1971) have identified counterrotating streamwise vortices in the wall region $\left(7 \leq y^{+}\right.$ $\leq 70)$ inclined at small angles $\left(\approx 3\right.$ to $\left.7^{\circ}\right)$ downstream away from the wall. Similar experiments (Kasagi et al. 1986, Smith and Schwartz 1983) have given similar evidence and identified that the region $14 \leq y^{+} \leq 25$ contains the most energetic vortices. Additionally they have identified single vortices as well as counter-rotating pairs. It is also inferred in these studies that streamwise vortices are responsible for creating low-speed streaks and lifting them away from the wall (i.e. ejections). Although it has also been argued that the cause-and-effect be reversed, i.e. streaks create vortices. Sometimes spanwise vortices were observed downstream of streamwise vortices (Clark and Markland 1971), although the evidence of them being connected was far from conclusive.

Whilst the wall region is populated with quasistreamwise vortices, the outer region, where the flow is intermittent, appears to be dominated by spanwise vortices with streamwise scale of the order of the boundary layer thickness. The bulges of the turbulent/nonturbulent boundary are identified as spanwise vortices with irrotational flow moving over and around the them, sometimes deep into the boundary layer, and being entrained.

HORSESHOE/HAIRPIN VORTICES

One of the earliest descriptions of important structures in turbulent boundary layers being of a vortical nature was given by Theodorsen (1952). Theodorsen's model was of horseshoe vortices growing outwards from the wall with heads inclined downstream at $45^{\circ}$ and spanwise dimensions proportional to the distance from the wall, as shown in figure 3.

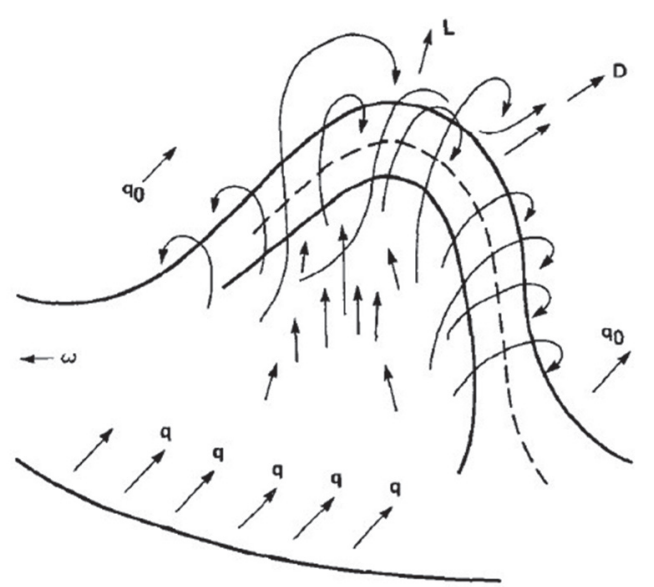

Figure 3 - Suggested horseshoe vortex model of wall-bounded turbulence (from Theodorsen 1952).

This model was developed from the vorticity transport form of the Navier-Stokes equations, and is based on the idea (with some physical reasoning) that initial concentrations of vorticity are enhanced by vortex stretching to create the origins of turbulence. Assuming that the wall-normal velocity gradient $(\partial U / \partial y)$ is greater by far than any of the other velocity derivatives (generally a reasonable assumption for wall-bounded flows) the vortex stretching term is simply that in equation 1 (where $\omega$ indicates vorticity).

$$
\omega_{x} \omega_{y} \frac{\partial u}{\partial y}
$$


This would be a maximum when the component of the vorticity vector in the $x-y$ plane is inclined at $45^{\circ}$ to the flow direction. The reason the horseshoe vortices should orientate themselves at the angle that makes their turbulence production a maximum is explained by Head and Bandyopadhyay (1981). It describes how the angle of the vortex is the result of the balance between the effect of the shear flow (which will rotate the vortex back towards the wall) and the induced velocity that each 'limb' of the vortex imposes on the other. Assuming that the vortex initially exists at an angle greater than $45^{\circ}$, as the vortex rotates towards the wall the rate of stretching will increase, and hence so will the induced velocity, tending to increase the angle of the vortex. Therefore, as long as the vortex is maintained at an angle greater than $45^{\circ}$, the situation remains stable. Should a vortex rotate past $45^{\circ}$ (or simply exist at a lesser angle) however, as the angle decreases the rate of stretching decreases, thereby removing the mechanism by which the vortex can maintain itself, leading to its dissipation and increasing rotation towards the wall.

This explanation is far from the only aspect of boundary layer structure that Head and Bandyopadhyay illuminated with their experiments. As they saw it themselves, the essential feature of their work was, "the existence of vortex pairs, or hairpin vortices, extending through at least a substantial part of the boundary-layer thickness, and inclined at a more or less constant characteristic angle to the surface" (Head and Bandyopadhyay 1981). This doesn't seem very different from Theodorsen's (1952) ideas that were thirty-years old at the time. The difference was in the approach. Whereas Theodorsen's proposal (1952) was a rather appealing intuitive hypothesis, the ideas that Head and Bandyopadhyay presented were, "forced upon the authors by the weight of the evidence that has accumulated in the course of the experiments" (Head and Bandyopadhyay 1981). Surprisingly, especially considering Theodorsen's (1952) earlier suggestions, Head and Bandyopadhyay claim that, "nothing could have seemed more implausible" (Head and Bandyopadhyay 1981) before they did their experiments.

Thereason the experiments revealed the structure to such effect was twofold. Firstly, as well as taking hot-wire measurements, which, while they are ideal for measuring the statistical nature of turbulence, are not very effective at revealing organised structures, they did flow visualisations by filling the boundary layer with smoke and illuminating it by an intense plane of light. At first the plane of light was orientated longitudinally (giving a side-on view of the flow) as shown in figure 4.

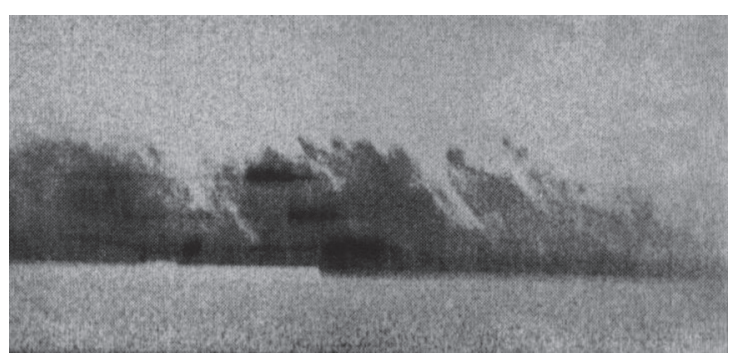

Figure 4 - Boundary layer structure at $R e_{\theta}=7500$ (adapted from Head and Bandyopadhyay 1981). Flow is from right to left.

These images, "suggested strongly that the edge of the layer at reasonably high Reynolds numbers was composed very largely of smokefilled features and smoke-free fissures inclined at a characteristic angle to the surface which appeared to be in the region of $45^{\circ}$ " (Head and Bandyopadhyay 1981). It was this that led to the second important experimental factor that made these experiments so revealing, the decision to, not only use a transverse light plane, but to incline this light plane at an angle of $45^{\circ}$ downstream and upstream (i.e. at $45^{\circ}$ and $135^{\circ}$ to the wall). The effect of this is best demonstrated pictorially, as in figure 5, but basically, with the light plane at $45^{\circ}$ downstream the vortex appears very briefly as the 
hairpin or horseshoe shape, whereas with the light plane at $45^{\circ}$ upstream the vortex cross-section gradually moves towards the wall as the vortex proceeds through the inclined light plane.

Figure 6 shows the resulting flow visualisations, and, although clearly the vortices are distorted from the idealised idea of the hairpin vortex, they are quite starkly present. The three pairs of images in figure 6 are at three different Reynolds
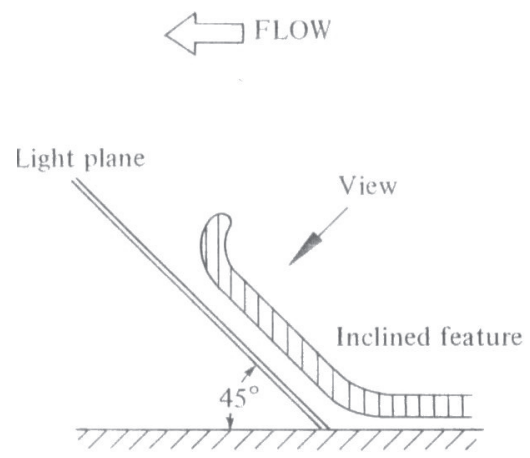

(a)

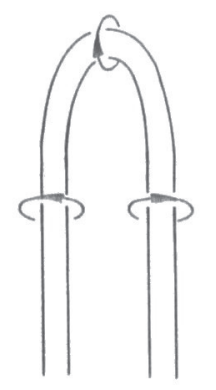

(a) numbers, $R e_{\theta}=600, R e_{\theta}=1700$ and $R e_{\theta}=9400$. This clearly shows the dependence of the scales of the vortices on the Reynolds number. Vortices can roughly be categorised as "loops" at very low Reynolds numbers, "horseshoes" at low-moderate Reynolds numbers, and "hairpins" at moderatehigh Reynolds numbers. A sketch from Robinson (1991) (after Head and Bandyopadhyay 1981) shows this geometry in figure 7 .

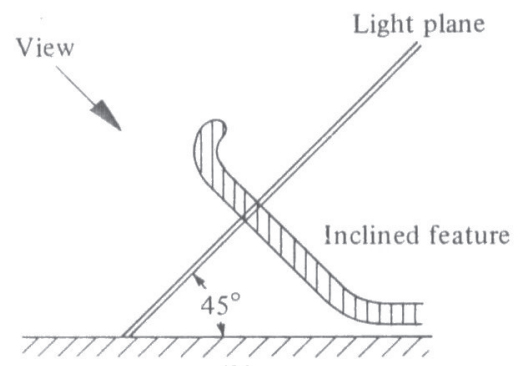

(b)

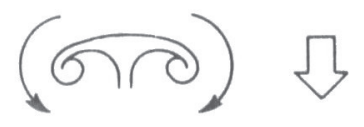

Apparent downward motion as feature convected past light plane

(b)

Figure 5 - Inclined features being convected past light plane at, (a) $45^{\circ}$ upstream, and (b) $45^{\circ}$ downstream, (from Head and Bandyopadhyay 1981).

Head and Bandyopadhyay (1981) were able to determine the length scale, $v / U \tau$, corresponding to their flow visualisations. Although a far from conclusive analysis, their comparison of this length scale (actually $100 v / U \tau$ ) to the cross-stream dimension of the hairpin vortices at all three Reynolds numbers does appear to show a close relationship between them, leading to the suggestion that the lateral dimensions of the vortices scale roughly with $v / U \tau$.

A final idea that Head and Bandyopadhyay (1981) suggested, based on what they saw in their experiments (in this case whilst using a longitudinal light plane), was that, "hairpins arise from the boundary in a regular sequence, with each hairpin as it leaves the surface giving rise to conditions that are favourable to the production of a further hairpin". This idea was born from the observation of a series of hairpins (at the characteristic $45^{\circ}$ to the wall) all lined up with their tips in a straight line (at a smaller angle to the wall $\approx 20^{\circ}$ ). This idea has a good degree of plausibility as a hairpin vortex will induce a velocity between its limbs upstream (creating a flow 

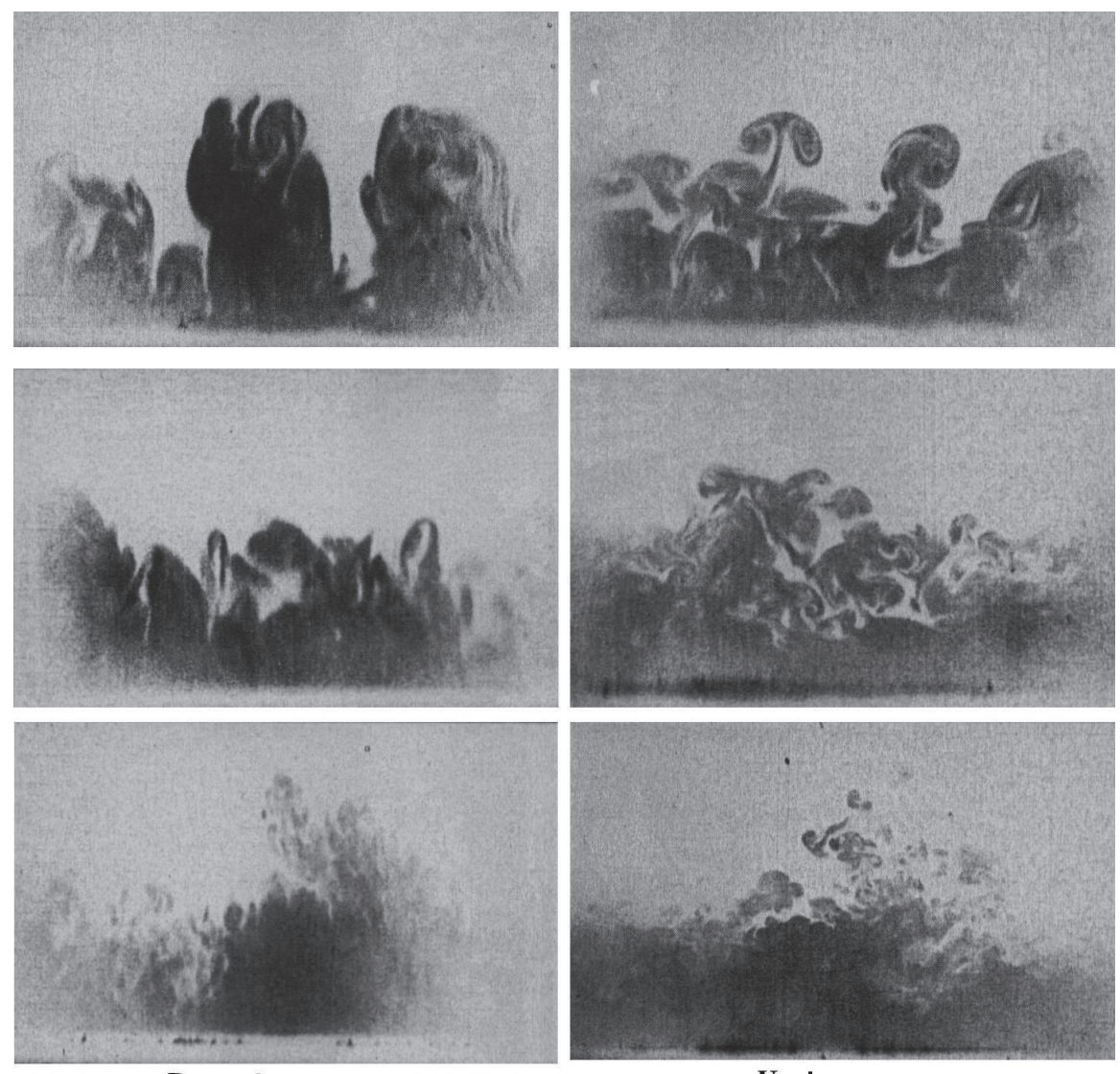

Downstream

Upstream

Figure 6 - Views with transverse light plane at $45^{\circ}$ upstream and downstream. From top: $R e_{\theta}=600, R e_{\theta}=1700$ and $R e_{\theta}=9400$, (adapted from Head and Bandyopadhyay).

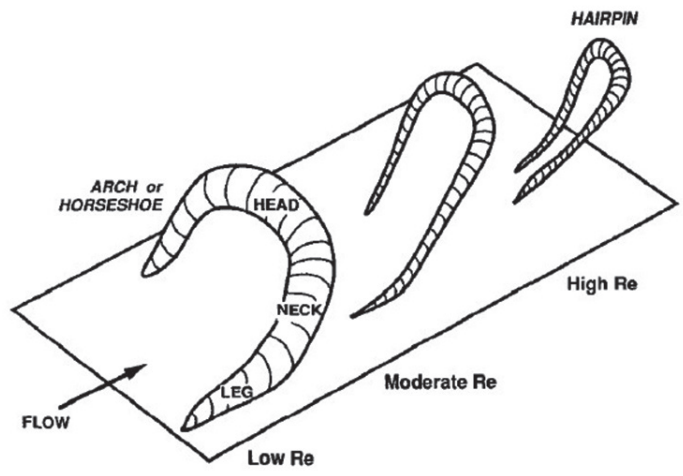

Figure 7 - Geometry of horseshoe/hairpin vortices (from Robinson after Head and Bandyopadhyay 1981).

deceleration in the immediate vicinity of the wall) and away from the wall, which could feasibly cause the creation of another hairpin vortex. It is noted that these agglomerations, although seen fairly rarely overall, were particularly noticed at higher Reynolds numbers (up to $R e_{\theta}=17,500$ ), which could indicate increasing commonality with increasing Reynolds number.

Although the results of Head and Bandyopadhyay (1981) indicate the presence of hairpins in a turbulent boundary layer, there is no ability to visualise and characterise a single hairpin, or track its development in time. This is partly due to the method of visualisation, but mainly due to the turbulent nature of the boundary layer, that makes it extremely difficult to focus on individual hairpins. To avoid this problem Haidari and Smith (1984) devised a study that, "examined the generation and growth of single hairpin vortices created by controlled injection of wall fluid from a streamwise slot with a laminar approach boundary layer". One of those single hairpins (marked with dye) is shown in figure 8. 


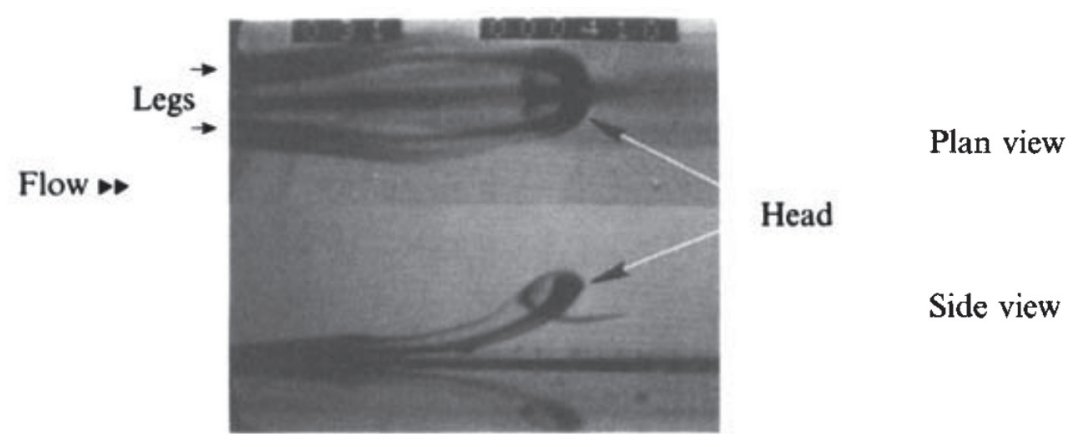

Figure 8 - Dual view of a dye-marked single hairpin vortex, (from Haidari and Smith 1994).

Haidari and Smith (1994) observed two scenarios: one in which the single hairpin would, "remain a single vortex, generally decaying without displaying growth of the overal flow structure", and another where it would, "stimulate the development of ancillary hairpin vortices, causing the overall flow structure to continually grow and expand". The second of these lends support to Head and Bandyopadhyay's (1981) idea of hairpins forming in clusters. The manual creation of hairpins in an otherwise laminar boundary layer, does not prove that such hairpins would exist, and behave similarly in a turbulent boundary layer without inducement. However, Haidari and Smith (1994) claim that the repeatability of the hairpin production implies that the hairpin vortex is a generic flow structure that could be created by a perturbation in a turbulent flow. Moin and Kim (1982, 1985), Kim and Moin (1986) performed direct numerical simulations (DNS) and large eddy simulations (LES) of a turbulent channel flow and provided the first view of hairpin vortices in a wall-bounded flow that was not based on flow visualisation. Instantaneous vorticity vectors in a plane $45^{\circ}$ to the wall and three-dimensional vorticity lines provided strong evidence of the existence of "appreciable numbers" of hairpin vortices (Moin and Kim 1985). Ensemble-averaged vorticity fields showed distinct horseshoe/hairpin shapes corresponding to to sweep and ejection events (Kim and Moin 1986). Thus, the presence of hairpin vortices was supported quantitatively in both instantaneous and ensemble-averaged vorticity fields. Obviously having three-dimensional quantitative measurements of hairpin vortices was a great advancement. Not long afterwards, particle image velocimetry was developed as an experimental technique for the measurement of turbulent flows. This provided two dimensional data from real flows at higher Reynolds numbers (Liu et al. 1991) and would go on to be used extensively to investigate coherent structures in turbulent wall-bounded flow. In particular, it lead to the notion of hairpin vortex packets, which is discussed in detail in section 4. One aspect of coherent structure identification that has proved difficult when vortices are discussed is the appropriate quantitative measure of vortices. This will not be discussed in this review, instead the interested reader is directed to the following references: Chong et al. 1990, Hunt et al. 1988, Jeong and Hussain 1995, Zhou et al. 1999, Chakraborty et al. 2005.

A further (much more recent) DNS study of a spatially developing boundary layer by $\mathrm{Wu}$ and Moin (2009) produced stunning visualisations of a forest of hairpin vortices filling the boundary layer as shown in figure 9. Rather reminiscent of Head and Bandyopadhyay (1981) the most striking aspect is perhaps the prevalence of the archetypal hairpin vortex shape. This is strong support for the hairpin vortex being a coherent structure of fundamental importance in wall-bounded turbulence. However, 


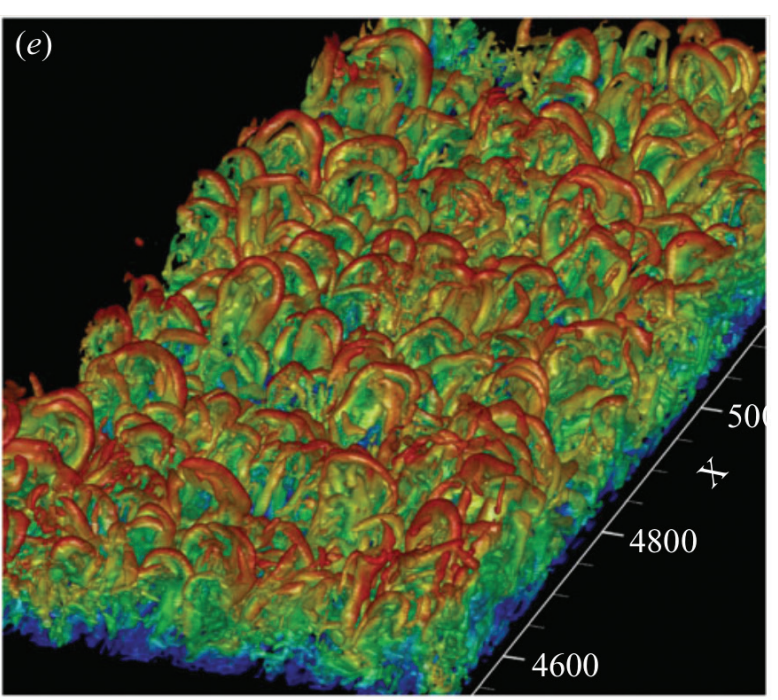

Figure 9 - Iso-surfaces of the second invariant of the velocity gradient tensor (from $\mathrm{Wu}$ and Moin 2009). The iso-surfaces are coloured based on local values of $U$ with higher $U$ represented by red.

it is important to note the low Reynolds number of this simulation $\left(R e_{\theta} \approx 900\right)$, and consider that such immaculate hairpins would be less likely to survive unspoilt at higher Re. This caveat is reinforced by the DNS Jimenez et al. (2006), which was approximately double the Reynolds number and show a much more complex forest of structures than simple hairpins as seen in figure 10. The authors describe the features of their forest as being, "oriented randomly, rather than with the predominant shear". Although they also state that many of them do resemble hairpins and indeed they found one instance of a 'train' of three hairpins, which could be interpreted as a 'packet', something we will return to in detail in $\S 4$. Certainly, comparing figures 9 and 10 gives the impression that the complexity of the vortical structures increases rapidly with $R e$ and quickly departs from the simple hairpin shape.

\section{VORTEX-BASED MODELS}

Due to the influence of the work discussed in $\S 2$, vortices have become the staple of many models used to describe wall-bounded turbulence. In this

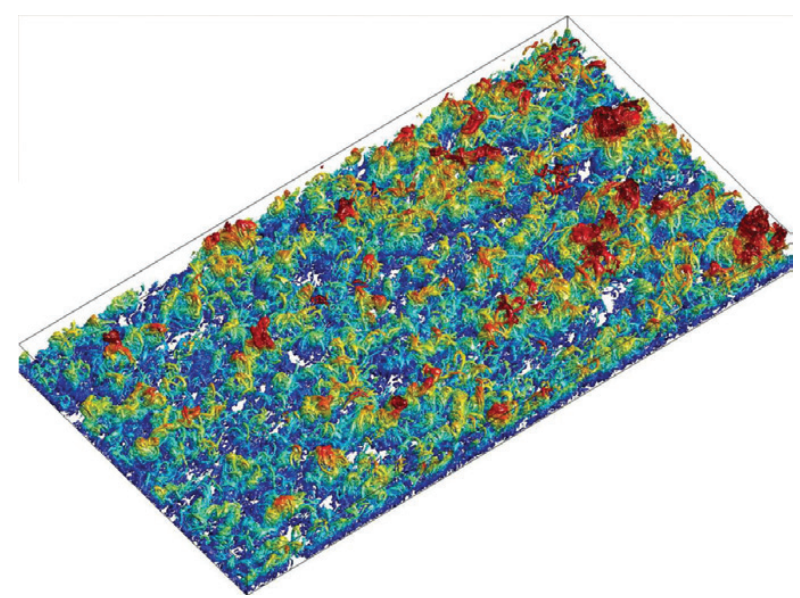

Figure 10 - Isosurface of the discriminant of the velocity gradient tensor from the simulation of Jimenez et al. 2010. The flow is from left to right, and the wall-parallel dimensions of the box are approximately $18 \times 9$ times the boundary-layer thickness at the centre of the box, spanning $R e_{\theta}=1420-1900$. The isosurface is coloured by the distance to the wall, from $y / \delta \approx 0.3-0.4$ for the deepest blue, to $y \approx \delta$ for the brightest red.

section some of the more interesting and insightful conceptual models are reviewed in what is a largely historical overview. It is interesting to revisit these models in view of our current understanding (which will be discussed in detail in $\S 4$ and §5) and take inspiration from the incredible insights some of them represent given the relatively meagre data the researchers possessed at the time.

\section{CONCEPTUAL Models}

Willmarth and Tu (1967) proposed an average eddy structure model based on their spacetime correlations between wall pressure and velocity. They found that, "near the wall the contours of constant correlation pv show a swept back structure so that the eddies must be primarily oblique"(Willmarth and Tu 1967), and that, "The contours of constant correlation pw in planes normal to the wall show an oblique disturbance moving away from the wall" (Willmarth and Tu 1967). They took these discoveries, combined with an analogy to the requirement of streamwise vortex components to produce the vertical motion of the 
spanwise vorticity in transition from laminar to turbulent boundary layer, to deduce, "a streamwise vortex component, together with the other two components of a three-dimensional vortex line of a hair-pin shape, also exists near the wall in the turbulent boundary layer and is an important part of the physical mechanism which maintains turbulence" (Willmarth and Tu 1967). This leap is based on the idea that the initially two-dimensional transverse vorticity lines are being stretched by the mean circulation and taking the form shown in figure 11, which can best be described as three- dimensional hairpin shapes, with a dominant streamwise component, sloped downstream at a shallow angle. Another idea that involved horseshoe vortices came from Black (1968). The simple idea was that vortices were "shed" from a near-wall instability, initially as closed loops (as shown in figure 12), but develop into horseshoe vortices by the evolution of the heads and upper portions of the vortices outwards and downstream, thereby stretching the trailing legs and inclining the vortex, whilst leaving the transverse element adjacent to the wall to decay in the viscous sublayer.

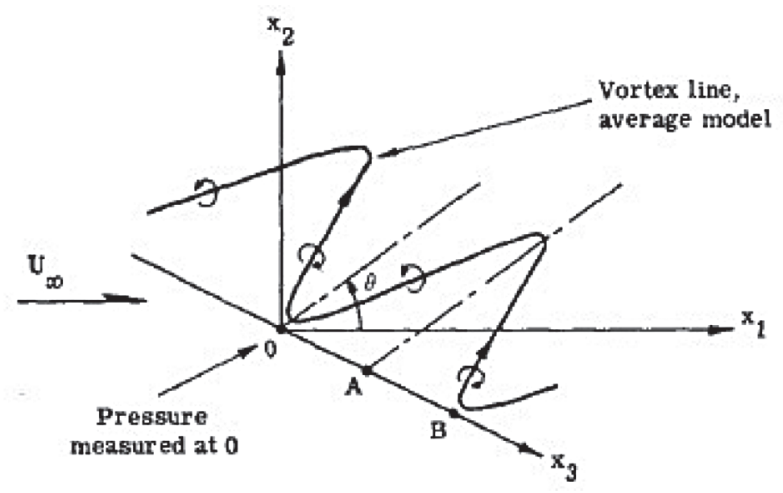

Figure 11 - Structure of an average model of vortex line near the wall (from Willmarth and Tu 1967).

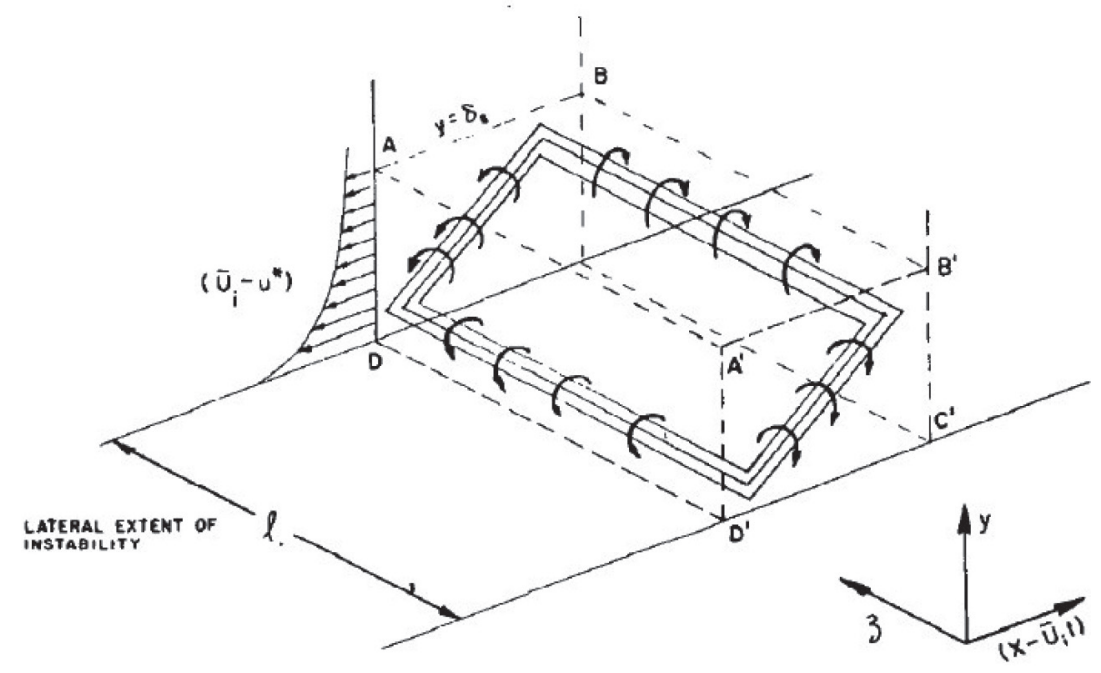

Figure 12 - Generation of ring vortices by instability in actual shear layer (from Black 1968). 
An important factor of Black's model was the idea that turbulent structure consisted of several horseshoe elements (in various stages of growth) that have a common trajectory in space. This is shown in figure 13, and is not too dissimilar to (and actually pre-dates) Head \& Bandyopadyay's observations that were discussed earlier (1981), although it is clear that figure 13 does not imply the $20^{\circ}$ agglomeration angle that Head and Bandyopadyay asserted. Black's concept was related to experimental results by suggesting that the vortex structures were inducing an outflow of low-speed fluid from within the vortex loop, which were being detected as sharp, intermittent spikes of Reynolds shear stress by stationary probes. Thus the random variation in size and strength of vortices is being used to explain intermittency.

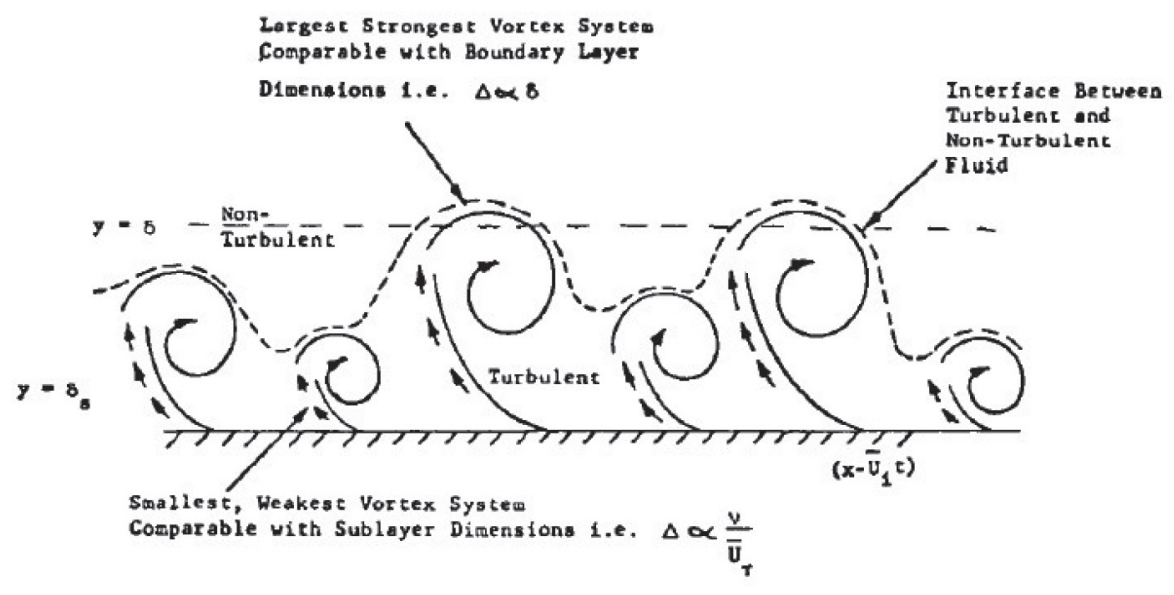

Figure 13 - Intermittency explained by random variation in strength of consecutive vortex systems (from Black 1968).

In a section of the second edition of his book, published in 1975, Hinze reviewed the various ideas and experiments regarding boundary layer structure and conceived a model that was an attempt to unify several of these elements into a single "cyclic"process. Hinze rightly points out that the model is only suggestive and not definite, but whilst it is slightly more complicated than most of the other concepts, it must be acclaimed for its completeness, in that it incorporates in some way almost all of the experimental observations of the time. The model is neatly summarised in figure 14, and although it is a cyclic process, and as such where one begins to describe it is immaterial, the following description will follow the order of the figure.

1. Large-scale disturbance (from the outer region or outer part of the wall region) causes a horseshoe vortex to be formed at the wall.
2. Vortex is deformed by the flow and becomes more elongated in the streamwise direction, i.e. tends towards becoming a hairpin vortex.

3. Tip of the loop moves away from the wall due to self-induction, entering regions of increasing velocity. Vorticity increases due to increased stretching

4. Vorticity causes outward flow between the legs of the vortex, causing local deceleration of fluid between the vortex and wall.

5. Ejection of low-momentum fluid away from the wall causes a positive contribution to the shear stress.

6. This causes a horizontal shear layer to be formed at around $5<y^{+}<30$, which shows as an inflectional dent in the instantaneous velocity profile (as mentioned earlier from Grass 1971) 


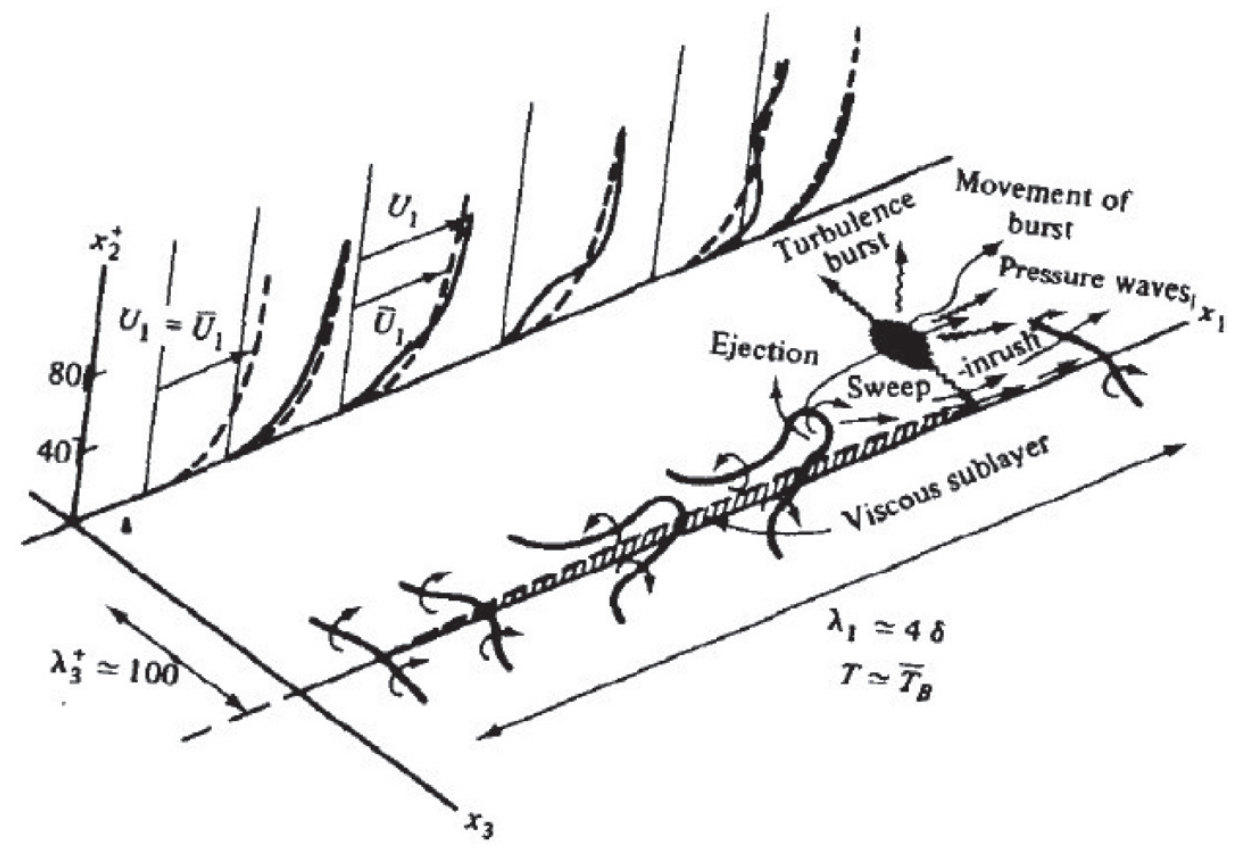

Figure 14 - Conceptual model of the turbulence near the wall during a "cyclic" process (from Hinze 1979)

7. The inflectional instability breaks down causing a turbulent burst.

8. Pressure waves propogate from the burst throughout the whole boundary layer.

9. "Blob" of fluid of high turbulence intensity is convected downstream and away from the wall, increasing in scale.

10. Pressure waves contribute (along with the negative $v$-component of velocity from the tip of the vortex loop before its breakdown) to a sweep/inrush flow towards the wall at an angle between $5^{\circ}$ and $15^{\circ}$ to the wall.

11. (The burst process, i.e. 7-10, makes a large contribution to the shear stress in the region $10<y^{+}<15$ ).

12. Horizontal sweep is retarded by the wall and contributes to birth of another horseshoe vortex (return to 1 ).

This model is highly idealised, and it can be argued that some of the linking arguments are weak, especially the latter stages of the cycle as it is described above. An example is that it is implicit in the model that the parent vortex is destroyed by the bursting process, although it is not clear why this should be the case. However, despite its ambiguities, it is quite insightful to see the instantaneous velocity profiles alongside the proposed mechanisms.

To a certain extent Hinze (1975) has neglected to (explicitly) relate his model to the presence of low-speed streaks. We can look to another concept, suggested by Smith (1984) after study of the literature and his own visualisations, as to how low-speed streaks and hairpin vortices are interlinked. The basic idea is that on top of a lowspeed streak there is an unstable shear layer, an oscillation occurs in this layer from which a vortex rolls-up and moves outwards (due to self-induction) and downstream (due to the velocity gradient). The low speed streaks themselves are created and maintained by the remnants of the hairpin legs, which remain near the wall and are stretched into counter-rotating quasistreamwise vortices, accumulating low-speed fluid between them as 
well as pumping fluid outwards (thus causing ejections). This all sounds somewhat familiar, but the further insight that Smith gives is shown in the upper part of figure 15 . Here the legs of the first hairpin vortex start to coalesce with the following vortex. This is important because the proliferation of this coalescence leading to the nesting of many consecutive hairpins could explain why low-speed streaks are observed to be much longer (about an order of magnitude) than quasi-streamwise vortices.

The lower diagram in figure 15 shows the coalescing hairpins from the side view at the characteristic angle of $45^{\circ}$ to the wall, as well as a nesting angle of between $15^{\circ}$ and $30^{\circ}$ (Head and Bandyopadyay (1981) suggested $\approx 20^{\circ}$ ).
Bernard and Wallace (2002) suggest a model, based on their reading of the literature, the main features of which are captured in figure 16. Here, the parent vortex is a quasistreamwise vortex that is attached to the wall at its upstream end and detached from the wall at its downstream end, the movement away from the wall increasing with downstream distance, to the point where the vortex sometimes turns into the spanwise direction. ("Attached" is defined as being within the viscous wall layer Bernard and Wallace 2002) Child vortices are said to appear around the point at which their parent detaches from the wall as quasistreamwise vortices with rotation opposite to that of the parent. This birth is as a result of the drop in spanwise velocity near the wall in the region of the parent's detachment.

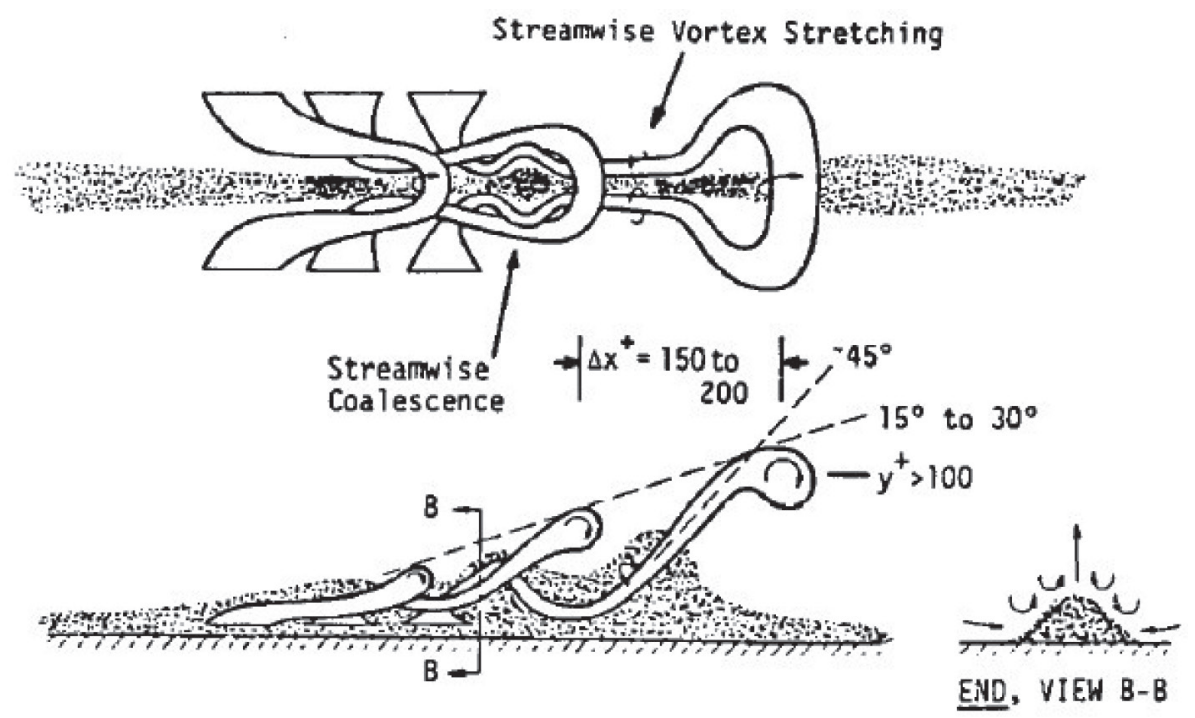

Figure 15 - Illustration of the breakdown and formation of hairpin vortices during a streakbursting process. Low-speed streak regions indicated by shading (from Smith 1984). Flow is from left to right.

The drop in spanwise velocity near the wall obviously occurs as the parent vortex moves further from the wall. The drop in velocity is like creating wall-normal vorticity, which is consequently reorientated into the streamwise direction through streamwise shearing. An alternative "child" vortex is the "arch vortex" also shown in figure 16. This is said to be created by the parent ejecting spanwise wall vorticity over a low-speed streak, from which the arch vortex rolls up.

This (Bernard and Wallace 2002) description of how the vortices develop has a lot in common with some of the previous models. Obviously the detached part of the parent convects downstream 


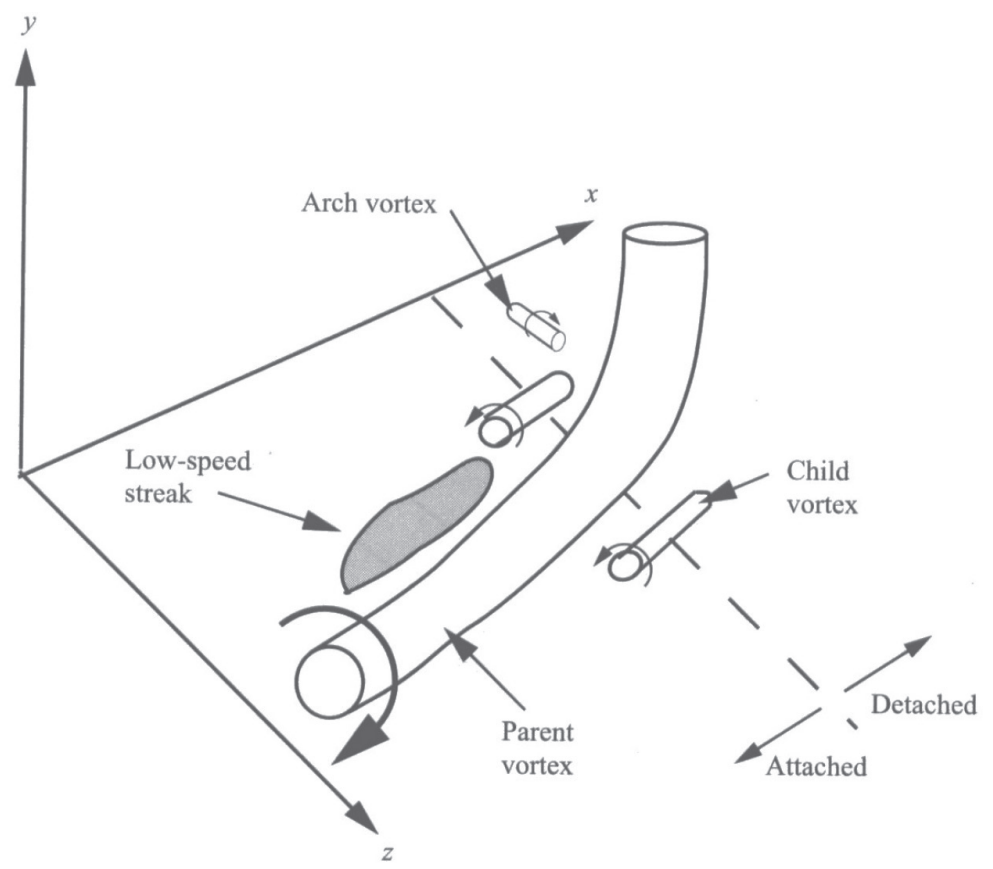

Figure 16 - Parent vortex showing where new streamwise and spanwise offspring are likely to arise, (from Bernard and Wallace 2002). Flow is in positive $x$ direction.

faster than the attached part due to the wall-normal velocity gradient. This parent is therefore stretched and hence strengthened. In tandem with its parent the child is also being stretched and strengthened as its downstream end detaches and rises from the wall, meanwhile the parent is busy adding new parts to the child at its upstream end in the region where its parent has detached. This leads to the situation in figure 17, which Bernard and Wallace (2002) took directly from Miyake et al. (1997). Here, the downstream end of the child vortex is situated above the upstream end of the parent vortex. Note that the vorticity of the child is always of the opposite sign to the parent.

Generally models involving coherent structures tend to allow slip at the wall. This can be justified by simply considering that the very thin viscous sub-layer allows for the flow to move relative to the wall (in other words anything below the sub-layer is neglected). Whilst this does not seem particularly unreasonable, one vortex-based model proposed by Moriconi (2009) did include the no-slip boundary condition. The kinematic model considered the streamwise velocity fluctuations induced by hairpin-like vortices and could reproduce the velocity profile and higher order statistics. If such a model could be extended to account for dynamical aspects it could potentially become genuinely predictive.

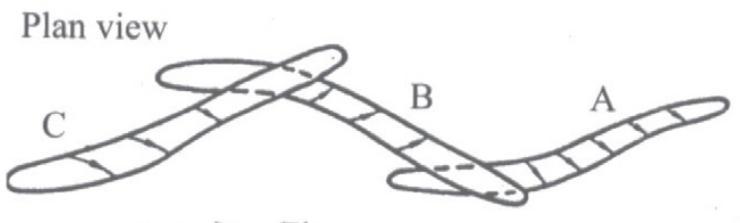

Flow

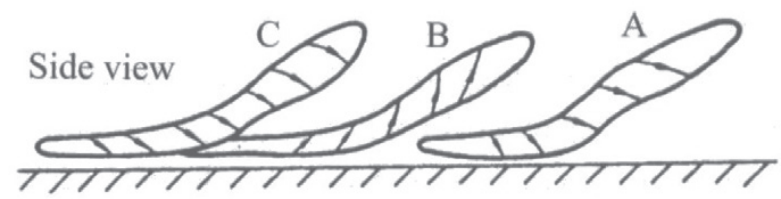

Figure 17 - Conceptual model of quasi-streamwise vortex structure; $\mathrm{A}$ is the parent of $\mathrm{B}$, which is the parent of C, (from Miyake et al. 1997). 
THE ATTACHED-EDDy ModeL

A model that is based on the "attached-eddy hypothesis" stands out from the other models mentioned so far as it has been used to mathematically predict certain aspects of wall-bounded turbulence. The attached-eddy hypothesis was originally proposed by Townsend in 1976. It was suggested that wall-bounded turbulence could be represented by arrays of eddies. The size of these eddies was said to be related to their distance from the wall, eddies further from the wall being larger, and hence their velocity fields extended to the wall. As such, the eddies were said to be attached to the wall. The hypothesis itself is that a random superposition of these attached eddies, of different sizes, but similar velocity distribution, can be used to describe the main energy-containing motion in a turbulent wall-bounded flow.
Townsend himself did not fully commit on the detail of the eddies themselves. He suggested that conical eddies were appropriate, but explicitly stated that there were other possibilities. The flow visualisations of Head and Bandyopadhyay (1981) (described earlier) showed the turbulent boundary layer to be populated by a forest of hairpin vortices inclined at 45 downstream to the wall. Perry and Chong (1982) noted that, "The $\Lambda$-vortex is a perfect candidate for Townsend's (1976) attached-eddy hypothesis". Throughout their work Perry and Chong always refer to hairpin/horseshoe vortices as $\Lambda$-vortices. In a way there is a distinction between the two as the $\Lambda$-vortex, shown in figure 18 , has a sharp point, but this is simply to make the mathematics of the model tractable and is an approximation to the hairpin vortex.

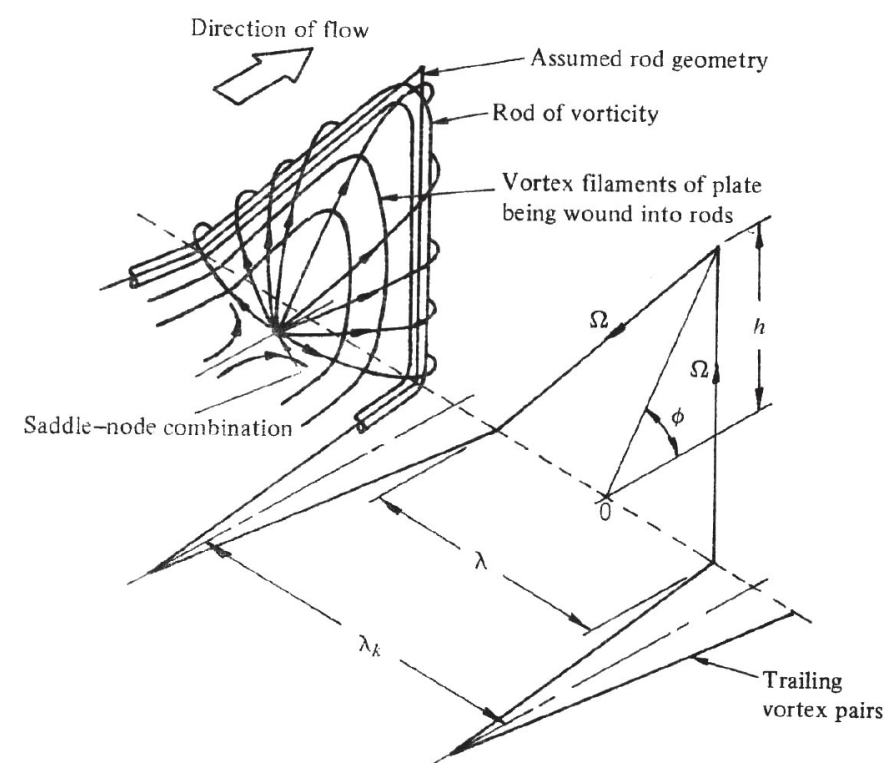

Figure 18 - $\Lambda$-vortex configuration (from Perry and Chong 1982).

Using figure 18 as a reference, it was shown that the $\Lambda$-vortex underwent a stretching process that increased its height $(h)$, whilst the distance between the legs of the vortex $(\lambda)$ decreased, such that, the product $(\lambda h)$ remained constant. When the legs of the $\Lambda$-vortex finally come together the vortex dies by vorticity cancellation.
It was also found that a range of scales of geometrically similar hierarchies must exist. A hierarchy is defined as a random array of $\Lambda$-vortices, all with the same circulation, but at different stages of stretching. It was suggested that the hierarchies formed by the viscous sublayer material rolling up (as shown in figure 19) to form the eddies of the 
smallest hierarchy, then, although most of these eddies die (by the mechanism described above), some pair up to form eddies of the next hierarchy. This continual process creates a hierarchy of geometrically similar eddies, responsible for the mean vorticity, Reynolds shear stress, and most of the energy-containing motions.

Initially the model could be used to predict the logarithmic law of the wall, a region of constant Reynolds shear stress, and an inverse-powerlaw spectral region for the fluctuating velocity components parallel to the wall in the turbulent wall region. Later, Perry et al. (1986) proposed that the attached eddies were surrounded by detached isotropic fine-scale eddies, said to be responsible for most of the turbulent energy dissipation. They also extended the model to include the wake region, where previously it had only been applicable to the turbulent wall region. The model continued to be developed and different eddy shapes were used, including $\Pi$ - shaped eddies, as the model was extended to be applicable to flows with a pressure gradient (Perry and Marusic 1995, Marusic and Perry 1995).

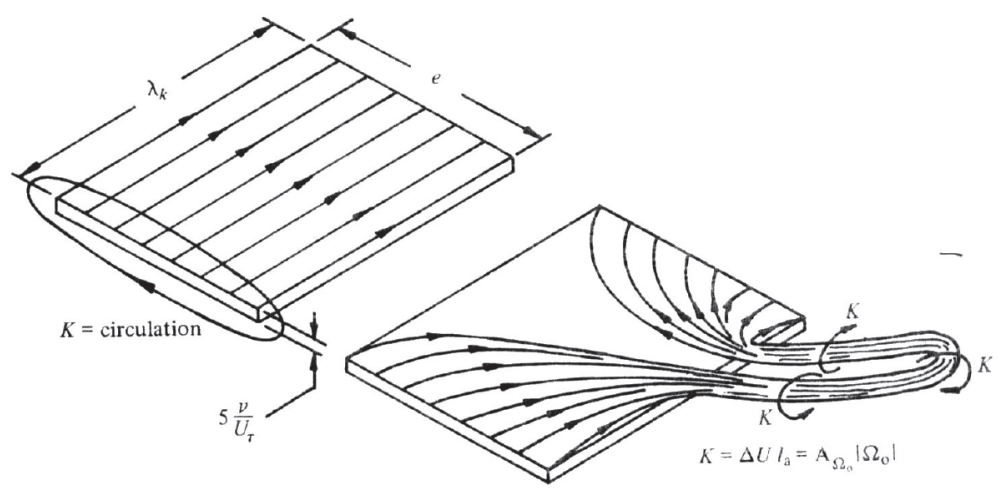

Figure 19 - Schematic of carpet of vorticity being wrapped into eddy, (from Perry and Chong 1982).

\section{HAIRPIN VORTEX PACKETS}

The features that Head and Bandyopadyay (1981) and Smith (1984) share, are the inspiration for one of the most widely accepted models. The hairpin vortex packet model was proposed by Adrian et al.(2000) as an attempt to unify disparate facts about turbulent boundary layer structure. The basic idea is that hairpin vortices are aligned coherently in the streamwise direction, with their heads in a distinct line inclining away from the wall at some angle, $\gamma$, (where $12^{\circ}<\gamma<20^{\circ}$ ), creating a larger-scale coherent motion called the hairpin vortex packet. The model states that hairpin packets originate from the wall from some (unspecified) disturbance that "creates a pool of low momentum at the wall" (Adrian et al. 2000). The primary vortex is formed and stretched by the velocity gradient (something that is familiar from other models) and grows continuously. If it is strong enough it will create a new upstream hairpin by inducing low speed fluid at the wall, that interacts with high speed fluid behind it. The secondary hairpin grows (by the same mechanism), and the process repeats. Apparently the primary hairpin is also capable of creating a hairpin downstream, which in turn can create more hairpins. In reference to the nesting angle, Adrian et al. (2000), say it depends on three factors:

1. The rate of continuous growth vertically (and spanwise).

2. The streamwise convection velocity of the hairpin.

3. The relatively uniform time between the formation of successive hairpins. 
Bringing in another observed feature of wallbounded flow, Adrian et al. (2000) suggest that low-speed regions form between the hairpins' legs. In a rare departure from Head and Bandyopadyay (1981) ideas, it is suggested that the hairpin legs are initially 50 viscous lengths apart and grow further apart with age. It was also argued that older (hence larger) hairpins move downstream faster than younger (smaller) hairpins because their induced back-velocity is lower. So as a packet ages the older hairpins move downstream faster than the younger hairpins in the same packet and the packet becomes stretched. (It should be noted that older hairpins, being larger, are more likely to be in faster moving fluid, i.e. further from the wall, which would also help them move downstream faster than younger, smaller hairpins closer to the wall.)

The hairpin packet model is summarised in figure 20, which shows three packets, each made up of several hairpins. The features (described above) of old and young packets are explicitly expressed, and the inset shows the nesting angle, $\gamma$ Note also the presence of two smaller hairpins in the outer region, suggesting the possibility of hairpins being formed locally away from the wall.

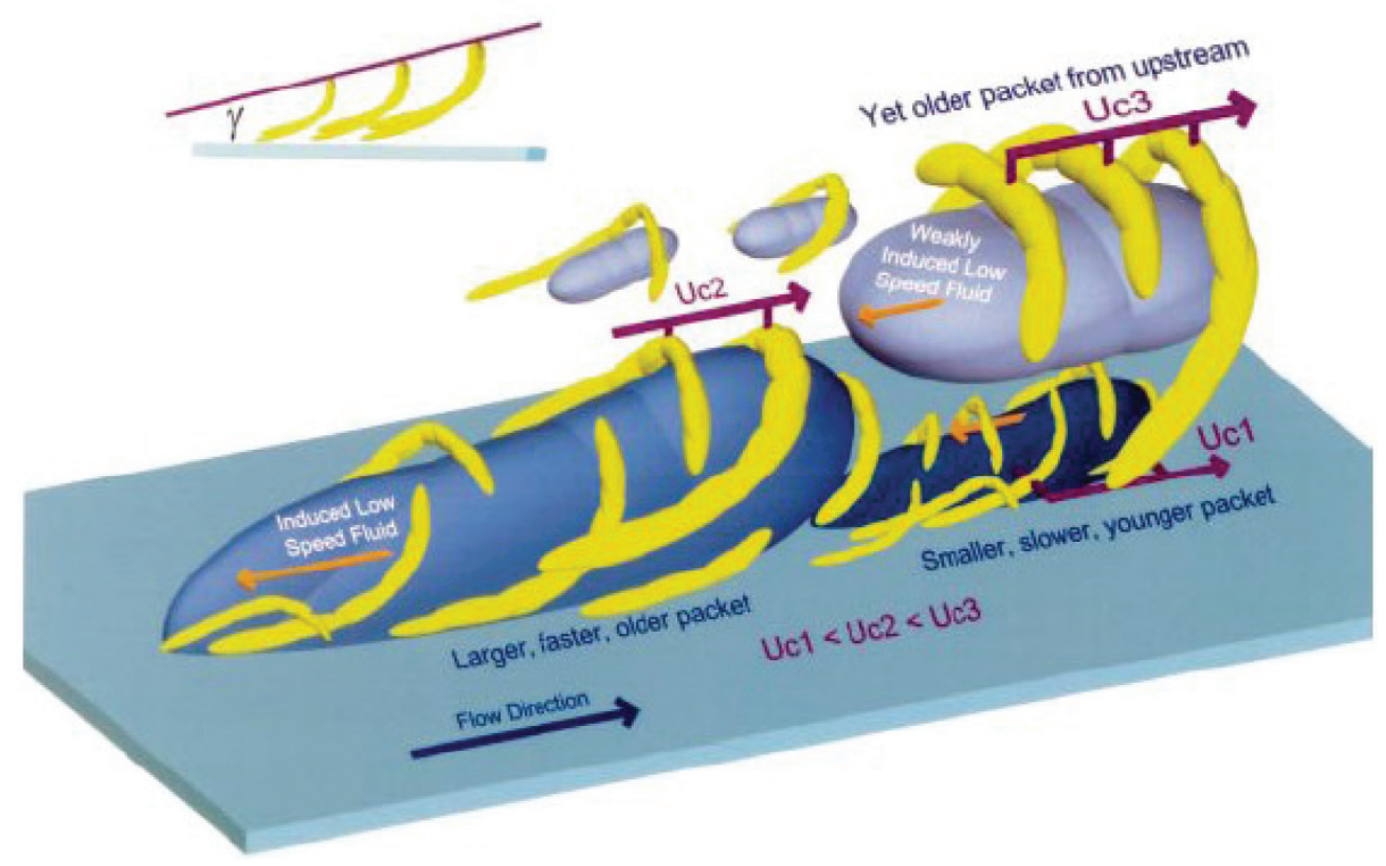

Figure 20 - Conceptual scenario of nested packets of hairpins aligned coherently in the streamwise direction (from Adrian et al. 2000).

A later investigation by Christensen and Adrian (2001), conjectured that if the vortex packet is a "dominant and robust" feature of wall-bounded turbulent flow, then it should leave an imprint on the statistics of the flow. Figure 21 shows instantaneous PIV results for two Reynolds numbers (a constant convection velocity has been removed to show the vortex structures). It shows numerous vortex cores, which are interpreted to be the heads of hairpin vortices, and a lifting of lowspeed fluid away from the wall under the upstream end of the hairpin head. Also, the nesting angle of the vortex packet is highlighted by the dashed line, and is seen to be $17^{\circ}$ and $16^{\circ}$, all of which is consistent with the hairpin packet model described above (Adrian et al. 2000). 


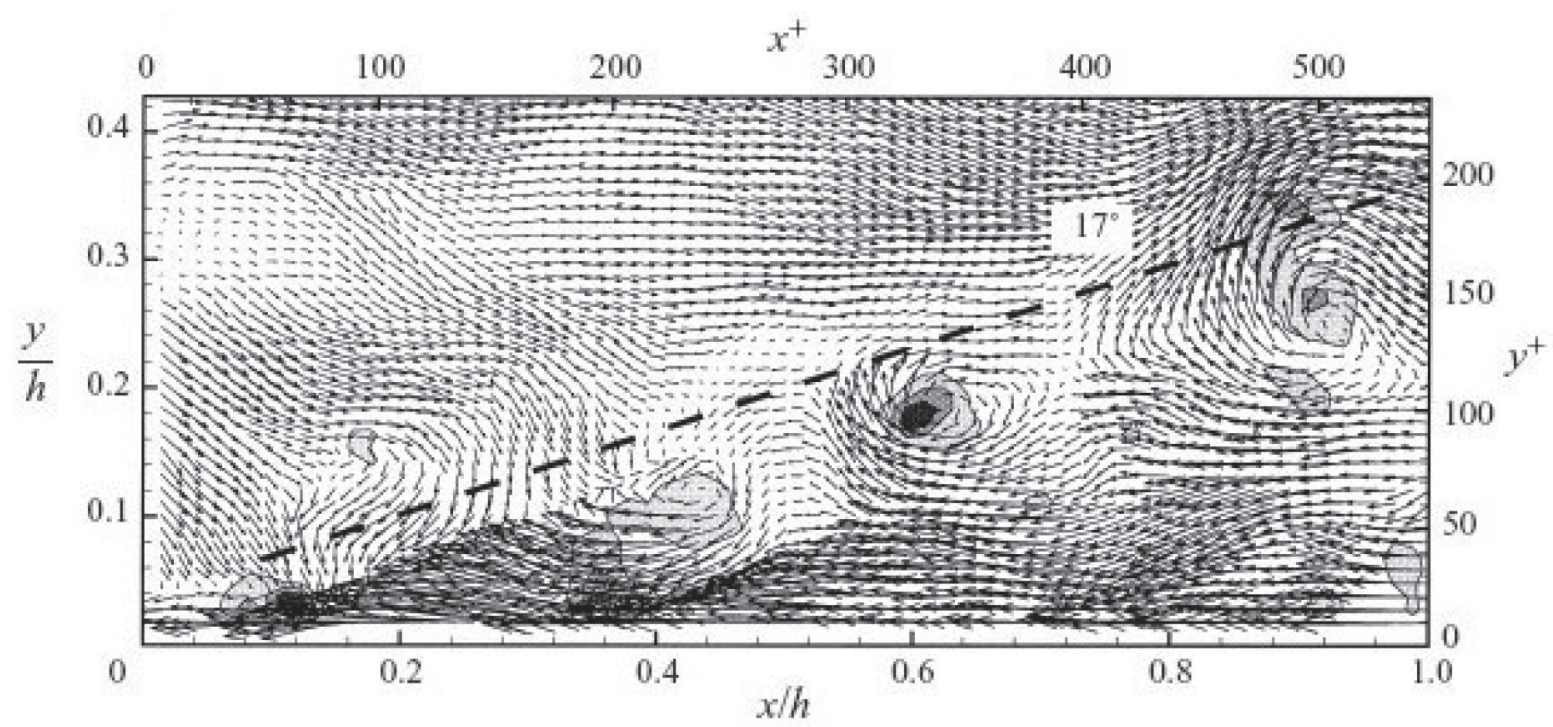

Figure 21 - Instantaneous velocity realizations in turbulent channel flow at $R e_{\tau}=547$, with a constant convection velocity removed, (from Christensen and Adrian 2000). Flow is from left to right.

Christensen and Adrian (2000) used two-point correlations between swirling strength and velocity to calculate a linear stochastic estimate (LSE) of the conditionally averaged velocity field. The results are shown in figure 22, where the length of all the vectors has been made unity by normalising them by their magnitude. The similarities between the stochastically estimated velocity fields (figure 22) and the instantaneous fields (figure 21) are clear, with the swirling motions A-C occurring, at similar intervals, on an incline angle consistent with the hairpin vortex packet model. The swirling patterns in figure 22 are quite clearly smeared in the streamwise direction. This is said to be due to the fluctuation of vortex spacing, and shows that the spacing between vortices is not regular.

Hambleton et al. (2006) also performed an LSE analysis of PIV data. However, in their experiments they used two orthogonal light-sheets and therefore captured the streamwise - spanwise and streamwise - wall-normal planes simultaneously. The results (shown in figure 23) from the streamwise - wallnormal plane appear very similar to those of
Christensen and Adrian (2001), in addition the simultaneously measured streamwise spanwise plane indicates the presence of two counter-rotating vortices commensurate with the presence of the legs of a hairpin.

Earlier work had identified the existence of uniform momentum zones in turbulent boundary layer flow and showed that they were rampshaped (Meinhart and Adrian 1995). Revisiting these results in light of the vortex packet paradigm provides an explanation for their existence in that they are the result of the induction effect in the interior of a vortex packet.

Zhou et al. $(1996,1999)$ investigated the matter of hairpin packet formation through simulation of a channel flow. An initial velocity field for the simulation is provided by linear stochastic estimation given a Q2 event (an event characterised by simultaneous $u<0$ and $v>0$ ), and resembles a hairpin vortex. In many aspects this is the simulated equivalent of Haidari and Smith's experiments discussed earlier (1994). The initial vortices that exceeded a threshold strength relative to the mean flow were found to evolve rapidly in the simulation, 


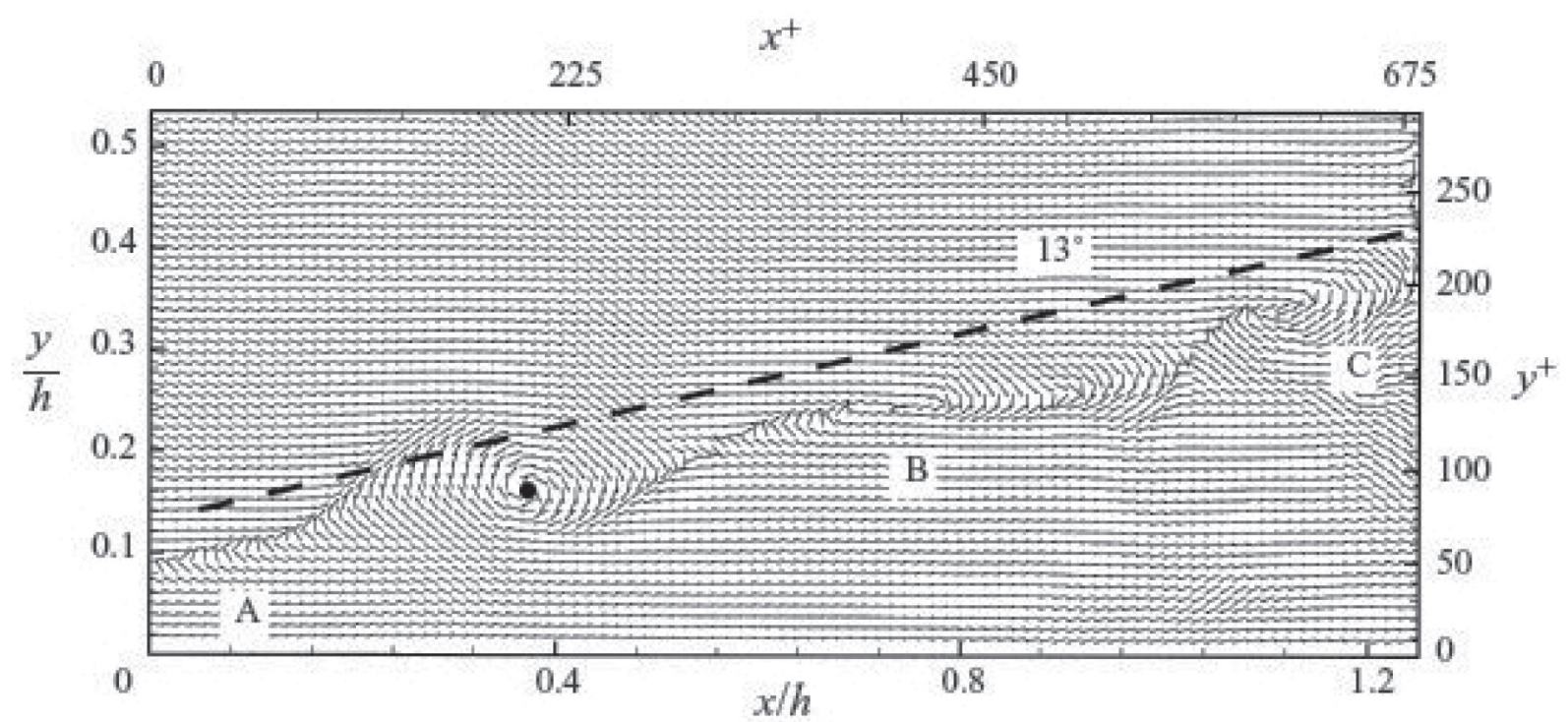

Figure 22 - Linear stochastic estimations of the conditionally average velocity field in channel flow at $R e_{\tau}=547$, (from Christensen and Adrian 2001). Flow is from left to right.
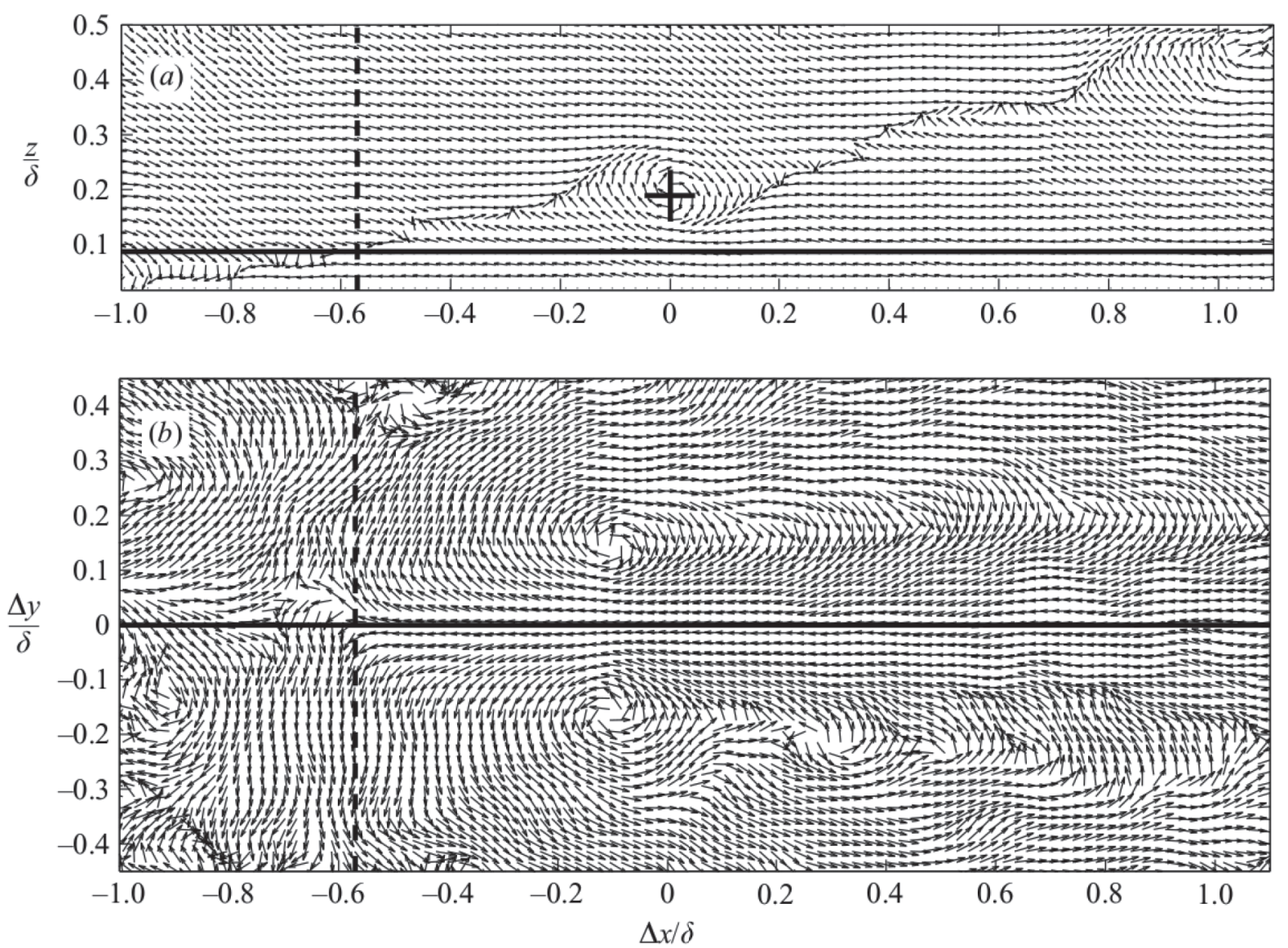

Figure 23 - Direction field from linear stochastic estimation of velocity based on positive signed swirl (swirl consistent with mean vorticity) at $Z_{r e f} / \delta=0.19$. (from Hambleton et al. 2006). The vertical plane is shown in (a), with the condition point marked with a cross, and the horizontal plane is shown in (b). Note that $y$ and $z$ are used for spanwise and wall-normal coordinates respectively (the opposite convention to this review). Flow is from left to right. 
if they didn't exceed the threshold they persisted as $\Omega$-shaped vortices before dissipating gradually. The evolution of those exceeding the threshold is briefly summarised by the following:

1. The primary hairpin vortex (PHV) develops into an $\Omega$-shaped vortex with trailing (quasistreamwise) legs, and continues to grow in all directions.

2. The PHV has small protrusions on its downstream end that develop into nearly streamwise vortices, and eventually roll up into an arch to form a downstream hairpin vortex (DHV).
3. Simultaneously, a secondary hairpin vortex (SHV) is created by the low-speed fluid that is pumped between the legs of the PHV and interacts with the high-speed fluid above the legs and leads to vortex roll-up.

4. The SHV then produces a tertiary hairpin vortex (THV) through the same mechanism as 3.

This autogeneration (as it is termed in Zhou et al. 1996) is therefore a mechanism by which hairpin vortices can reproduce, and thus can produce hairpin packets. The resulting hairpin vortex packet is shown in figure 24, with the vortices labelled according to the above description.

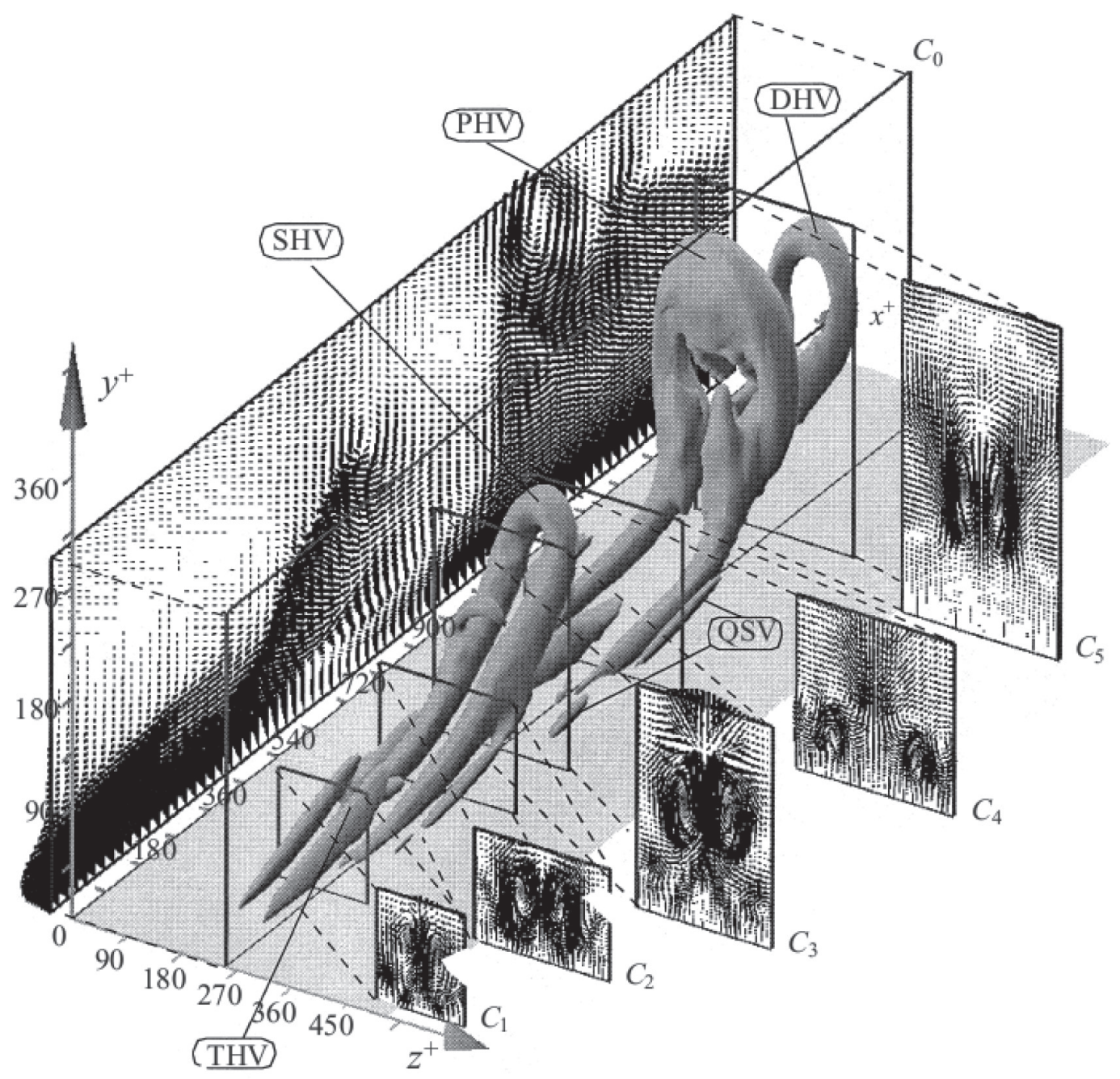

Figure 24 - The hairpin vortex packet produced by the simulations of Zhou et al. 1999. Flow is in positive $x$ direction. 
The perfect symmetry of the hairpins in figure 24 is said to be because the initial Q2 event has no spanwise velocity, and making this velocity non-zero in the intial state produces asymmetric 'cane' vortices (Zhou et al. 1999). Also, in Adrian and Liu (2002), a small amount of background noise was added to the simulation. This created a more chaotic packet, as shown in figure 25, but one that was still recognisably similar to that shown in figure 24. The packet involves some clear differences to the clean case, and in particular the upstream vortices (the SHV and THV) are far from ideal hairpin shapes. However the fact that the clean and noisy simulations both produce a vortex packet through autogeneration demonstrates the robustness of the autogeneration mechanism, which is a property it would certainly require to be consistent in turbulent flow. Not that these simulations can be considered proof that packet generation occurs in real turbulent flows.

Ganapathisubramani et al. (2003) investigated an $R e_{\theta}=2500$ turbulent boundary by performing stereoscopic particle image velocimetry measurements (SPIV) in the streamwise-spanwise plane (i.e. parallel to the wall). These results showed signatures of the vortex packets proposed by Adrian and co-workers: groups of hairpin vortices aligned in the streamwise direction. Planar results cannot provide a full picture of a hairpin, but in this case hairpins were interpreted in the results because, "usually, the hairpin vortices observed have two legs" (Ganapathisubramani et al. 2003), i.e. pairs of counter-rotating wall-normal vortices were observed, which is what one would observed if a hairpin was present with its legs crossing the measurement plane.

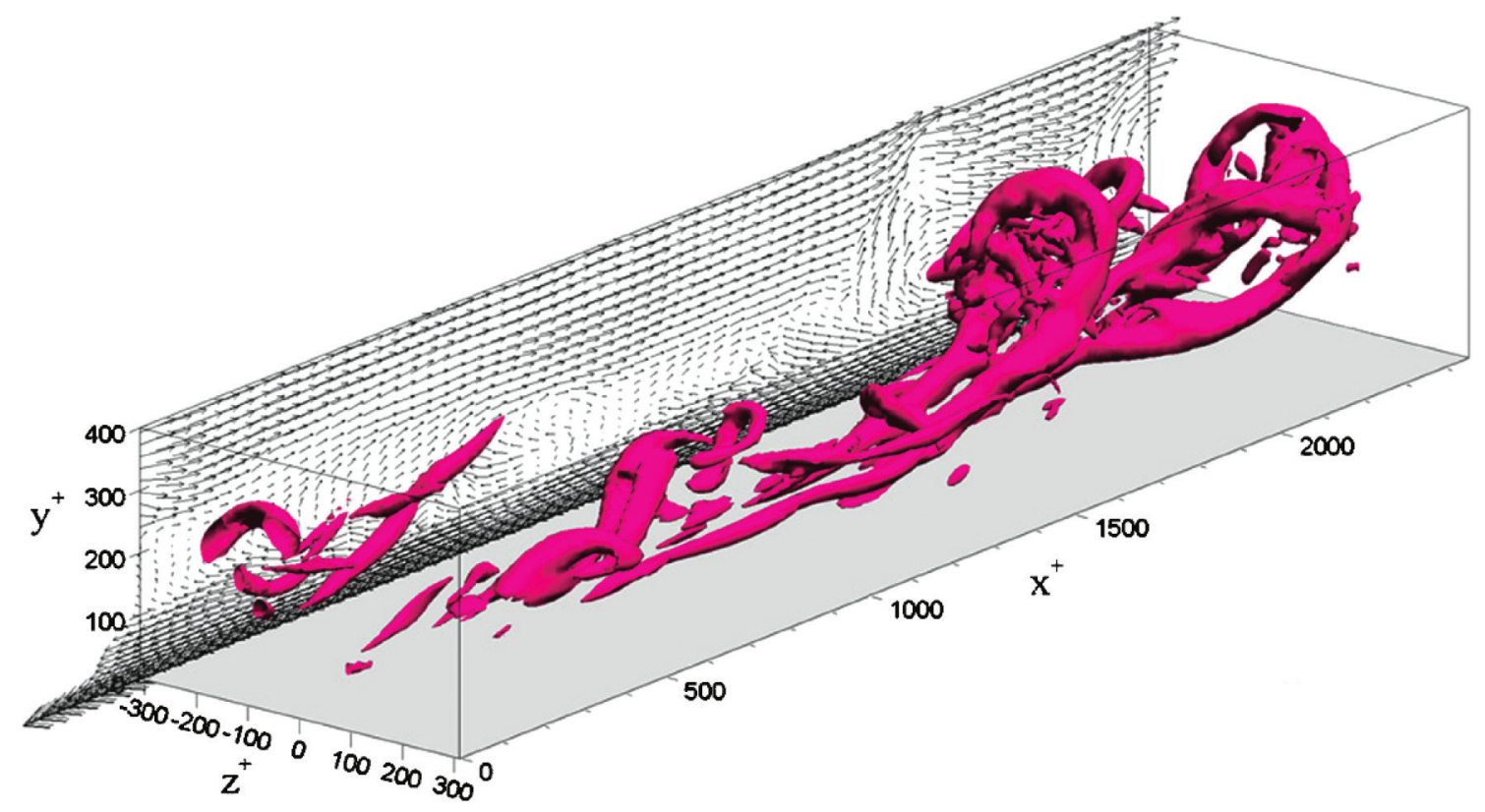

Figure 25 - The hairpin vortex packet produced by the simulations of Adrian and Liu et al. (2002) that include $5 \%$ noise, taken from Adrian 2007. Flow is in positive $x$ direction.

Dennis and Nickels (2011) performed high speed SPIV in a turbulent boundary layer at $R e_{\theta}=4700$. The laser sheet was orientated perpendicular to the mean flow (i.e. crossstream) so coherent structures present in the flow were convected through the measurement plane and could be effectively "scanned" as they did so. This technique coupled with the use of Taylor's hypotheses (1938) (which has been shown to 
be reasonable for this type of study (Dennis and Nickels 2008) enabled the production of pseudo$3 \mathrm{D}$ velocity fields, which showed the presence of flow structures closely resembling hairpin vortex packets, an example of which is shown in figure 26. The black iso-surface utilises the swirling strength to indicate the presence of vortices. There is a clear set (i.e. packet) of vortices, labelled in figure 26, that arch over the low speed region shown in blue. The first vortex is largest and most closely resembles an archetypal hairpin/horseshoe. This pseudo-instantaneous snapshot is highly reminiscent of the idealised hairpin vortex packet model schematic in figure 20. A very similar picture is seen in a supersonic (Mach 2) boundary layer at $R e_{\theta}=34000$ using tomographic PIV to obtain all three components of velocity in a small volume by Elsinga et al. (2010).

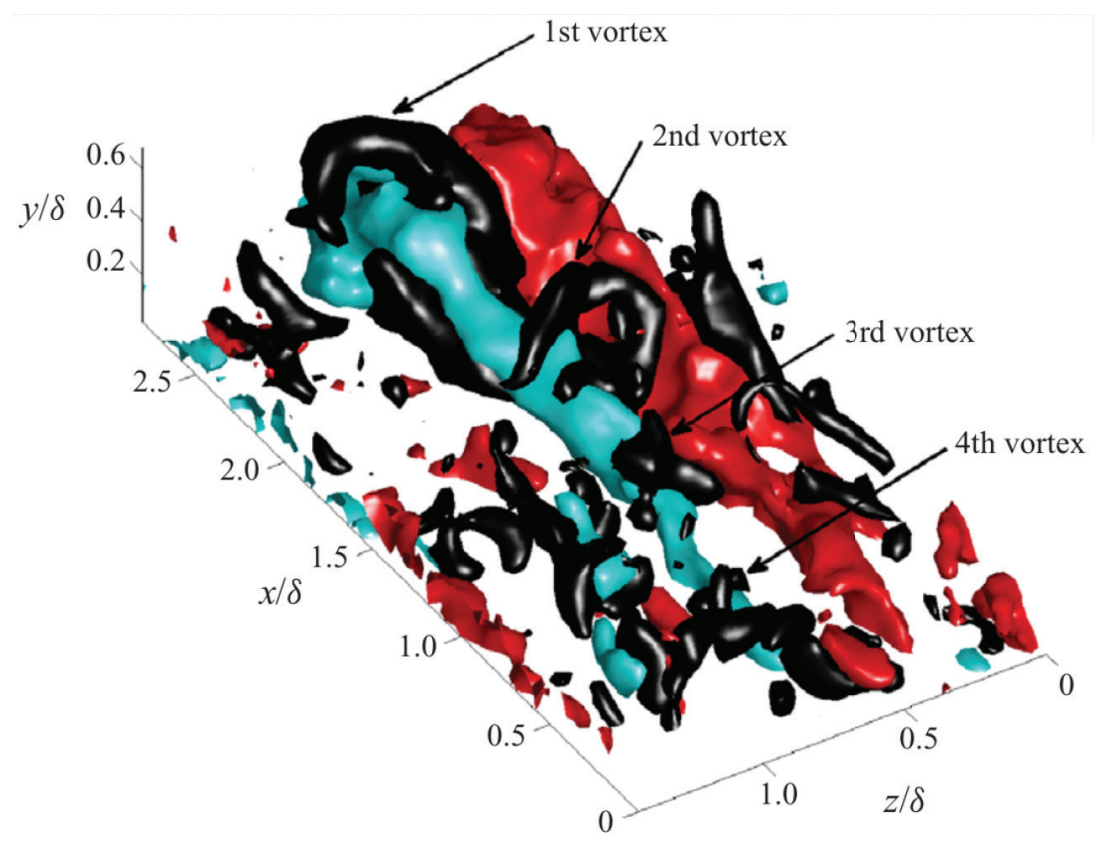

Figure 26 - Visualisation of a hairpin vortex packet with high- and low-speed structures (from Dennis and Nickels 2011). Black iso-surface: $\left|\lambda_{\text {ciliso }}\right|=0.18\left|\lambda_{\text {ci }}\right|_{\max }$. Blue iso-surface: $u_{i s o}=0.1 \bar{U}$. Red iso-surface: $u_{i s o}=0.1 \bar{U}$. Flow is in positive $x$ direction.

Using a conditional averaging technique Dennis and Nickels (2011) also demonstrated the average vortices for different distances from the wall as shown in figure 27. It is noticeable that the legs of all the vortices extend to the wall and that they scale with distance from the wall. The vortices closer to the wall present extra elements (sometimes referred to as "tongues"). This feature is reminiscent of the dye visualisations of Haidari and Smith (1995) (see figure 8), which show a downstream extension that is similar to that observed in these conditionally averaged fields. Also, in the simulations of Zhou (1999) they observed that, "in addition to the hairpin vortex, a pair of vortical tongues can be seen to stick out at the downstream end of the hairpin vortex,". In this case the tongues are seen to develop into another hairpin vortex and so the conditionally averaged results of Dennis and Nickels 2011 b could be taken as evidence indicating the robust presence of a hairpin vortex packet, i.e. on average a hairpin near the wall has another hairpin just downstream of it. 

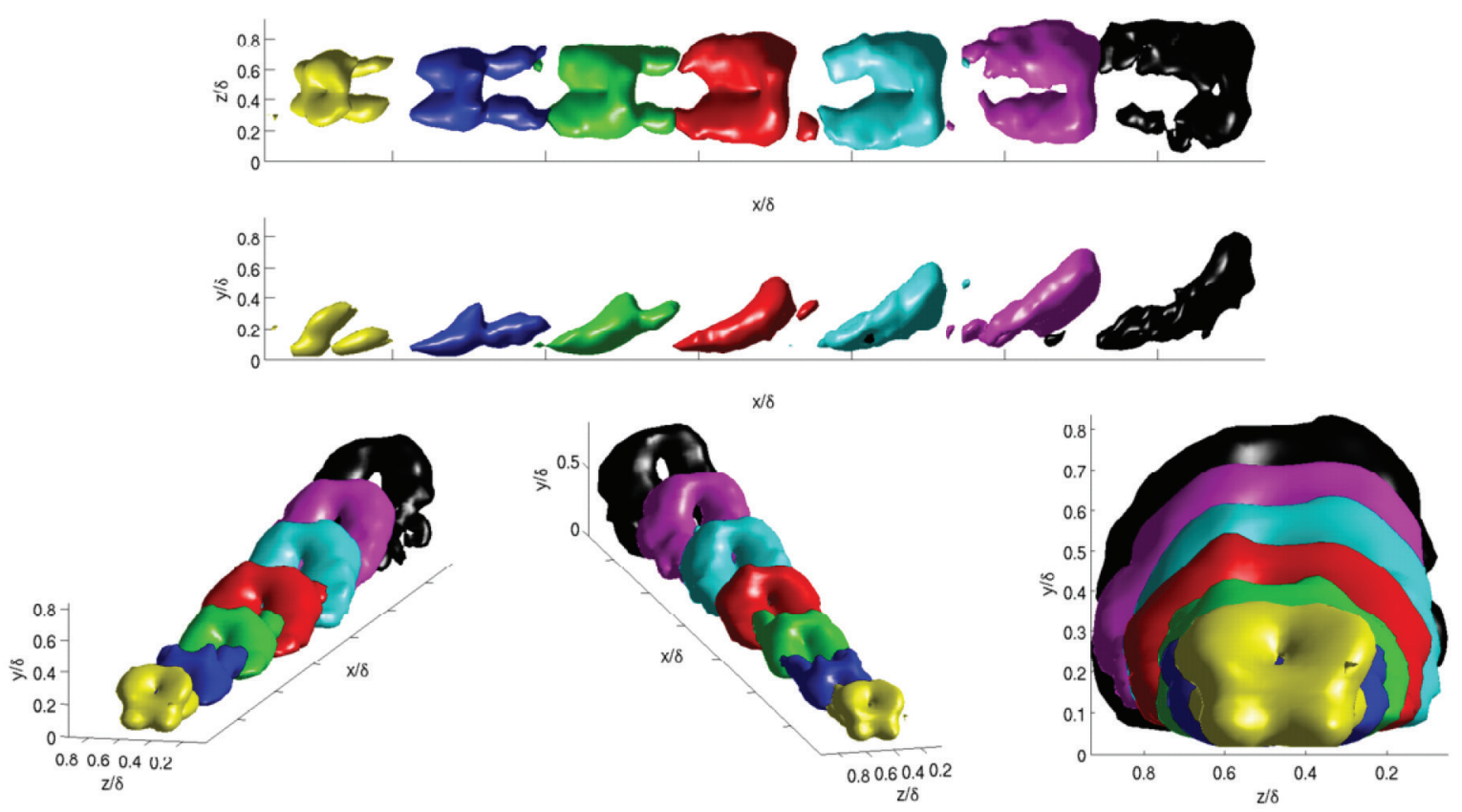

Figure 27 - Conditionally averaged swirling fields given spanwise swirl showing the variation in average vortex with distance from the wall (from Dennis and Nickels 2011a). Condition is for $\lambda_{c i z}>0.18 \lambda_{c i z, \max }$, iso-surfaces shown are for $\lambda_{c i, i s o}=0.08 \lambda_{c i, \max }$.

Although the term vortex "clusters" rather than packets is used by the authors, the DNS simulations of del Alamo et al. (2006) of a $R e_{\tau}=1900$ channel flow, also provide an overall picture that is rather similar to the vortex packet paradigm. In particular they study, "tall clusters rooted in the near-wall region" which are associated (on average) with a wall-normal ejection surrounded by two inclined counter-rotating vortices as shown in figure 28. One particular aspect that the authors note is that although the average flow pattern is consistent with a "single large-scale vortex loop" (a hairpin if we were inclined to call it such) the individual clusters seen in the DNS are markedly more complex. The observations of del Alamo et al. (2006) lead to the conclusion that the clusters cannot have grown from the wall (as they are too short-lived) but instead that clusters are created by larger clusters in front of them (a process which repeats self-similarly). Despite the different nomenclature, this idea of vortex clusters is very similar (kinematically at least) to the vortex packet model.
Further support for the hairpin vortex packet model was found by Marusic (2001). The attached-eddy model was extended to incorporate a packet of $\Pi$-shaped eddies. This procedure improved the results of the attached-eddy model by more accurately producing the streamwise elongation of the two-point spatial correlation found in experiments. The conclusion drawn was that spatially coherent packets of vortices are statistically significant.

One of the issues that surrounds the concept of hairpin vortices and vortex packets is the large number of seemingly disorganised vortical motions that surround them. The problem is particularly noticeable in DNS data as all scales are resolved, whereas often the experimental measurement technique acts as a low-pass filter on the data and removes some of the small-scale clutter. The issue is not just that the clutter makes the identification of vortex packets difficult, but that it is only likely to increase with increasing Reynolds number. This leaves the question of whether the vortex packet 


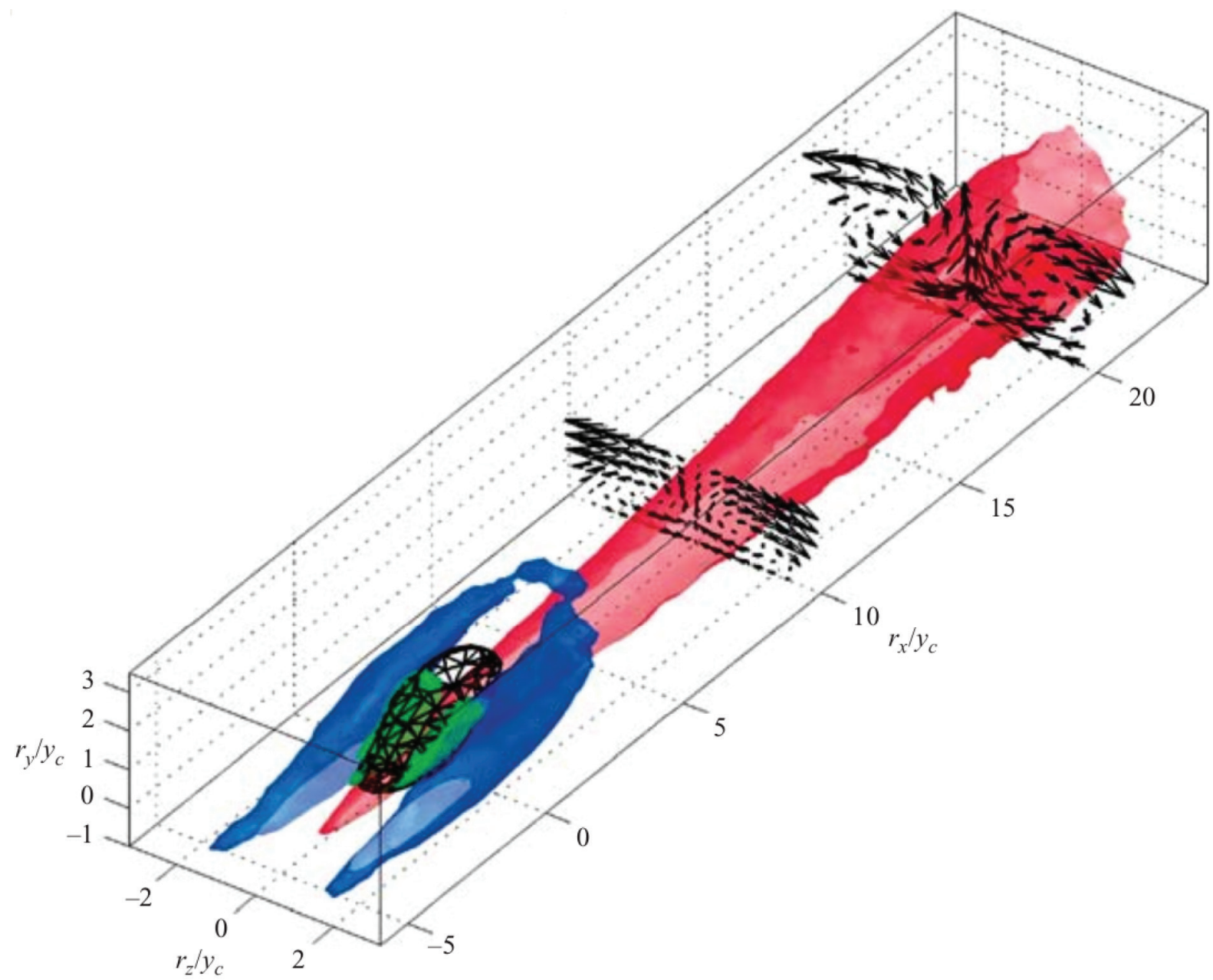

Figure 28 - Three-dimensional plot of the average velocity field conditioned to the tall attached clusters (taken from del Alamo et al. 2006). The black mesh is an isosurface of the p.d.f. of the vortex positions and contains $57 \%$ of the data. The blue volume surrounding the cluster is the isosurface $u^{+}=0.3$. The red volume downstream of the cluster is the isosurface $u^{+}=0.1$. The green volumes indicate the vortices (based on the discriminant criterion). Flow is in positive $r_{x}$ direction.

idea is something that can only be considered relevant to low to moderate Reynolds number flows. Clearly the difficulty of performing experiments at high $\operatorname{Re}$ (and the impossibility of doing it with DNS), means there is limited information available at high Re. However, one area that has been probed is the atmospheric boundary layer. Hommema and Adrian (2003) performed a smoke visualisation in an $R e_{\theta}>10^{6}$ atmospheric boundary layer. The results (shown in figure 29) show ramp-like structures that could be interpreted as the large-scale motions associated with hairpin vortex packets. They do, at least, look remarkably similar to the laboratory-scale flow visualisations and 2D PIV results discussed previously (see figures 4 and 21), which is an encouraging sign that the vortex packet concept is relevant to high Reynolds number flows.

\section{VERY LARGE-SCALE MOTIONS}

A structural element of wall-bounded turbulent flow that has recently received attention is the, "regime of verylong meandering positiveand negative streamwise velocity fluctuations" (Hutchins and Marusic 2007a), which Hutchins and Marusic (2007a) christened "superstructures", but are also commonly referred to as very large-scale motions (VLSMs). It has been 


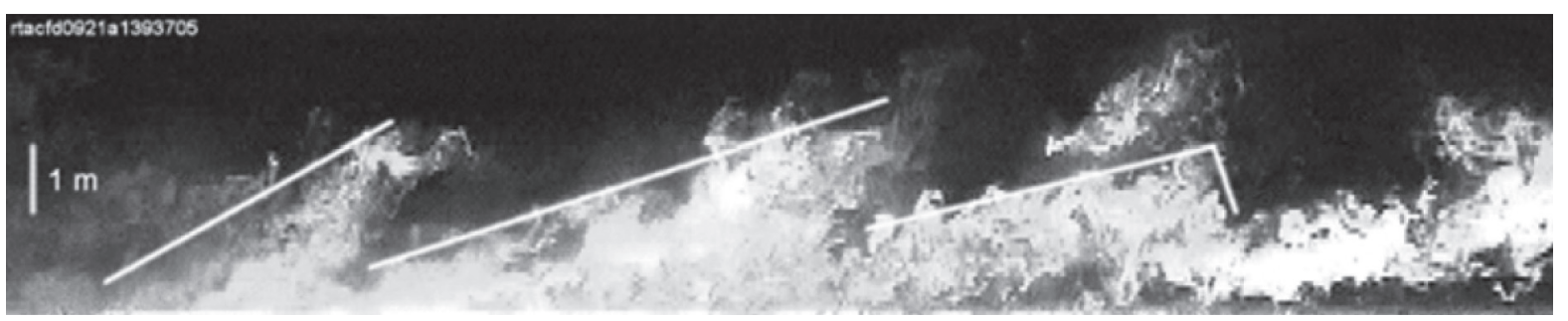

Figure 29 - Smoke visualization of the wall-region $(0<x / \delta=<0.01,0<\mathrm{y} / \delta<0.015)$ of the atmospheric boundary layer at $R e_{\theta}=9 \times 106$ (from Hommema and Adrian 2003). Flow is from left to right.

suggested that superstructures are found in boundary layers and VLSMs are found in pipe and channel flows. The distinction has been made because there appears to be some subtle differences between the very large structures in the boundary layer and the internal flows (which will be discussed in the following). However, the term VLSMs (not superstructures) will be used in this review as the structures are certainly very similar and don't really warrant different nomenclature (in the author's opinion).

VLSMs are found (unlike low-speed streaks) in the log and lower wake regions of the turbulent boundary layer. Figure 30 shows a sample from a hot-wire rake positioned at $y / \delta=0.15$ in a $R e_{\tau}=$ 14380 boundary layer flow. The $x$-dimension has been created by projecting temporal measurements using Taylor's hypothesis (1938). A very long feature can be seen meandering through the boundary layer for over $20 \delta$ (over $6.5 \mathrm{~m}$ at the experimental Reynolds number). This is compared to the PIV result shown in inset (b) in figure 30, which is said to represent a snapshot of the much longer structure, (a). The meandering nature of this structure is said to be the reason why its true length is not apparent to one-dimensional correlations, which is quite feasible.
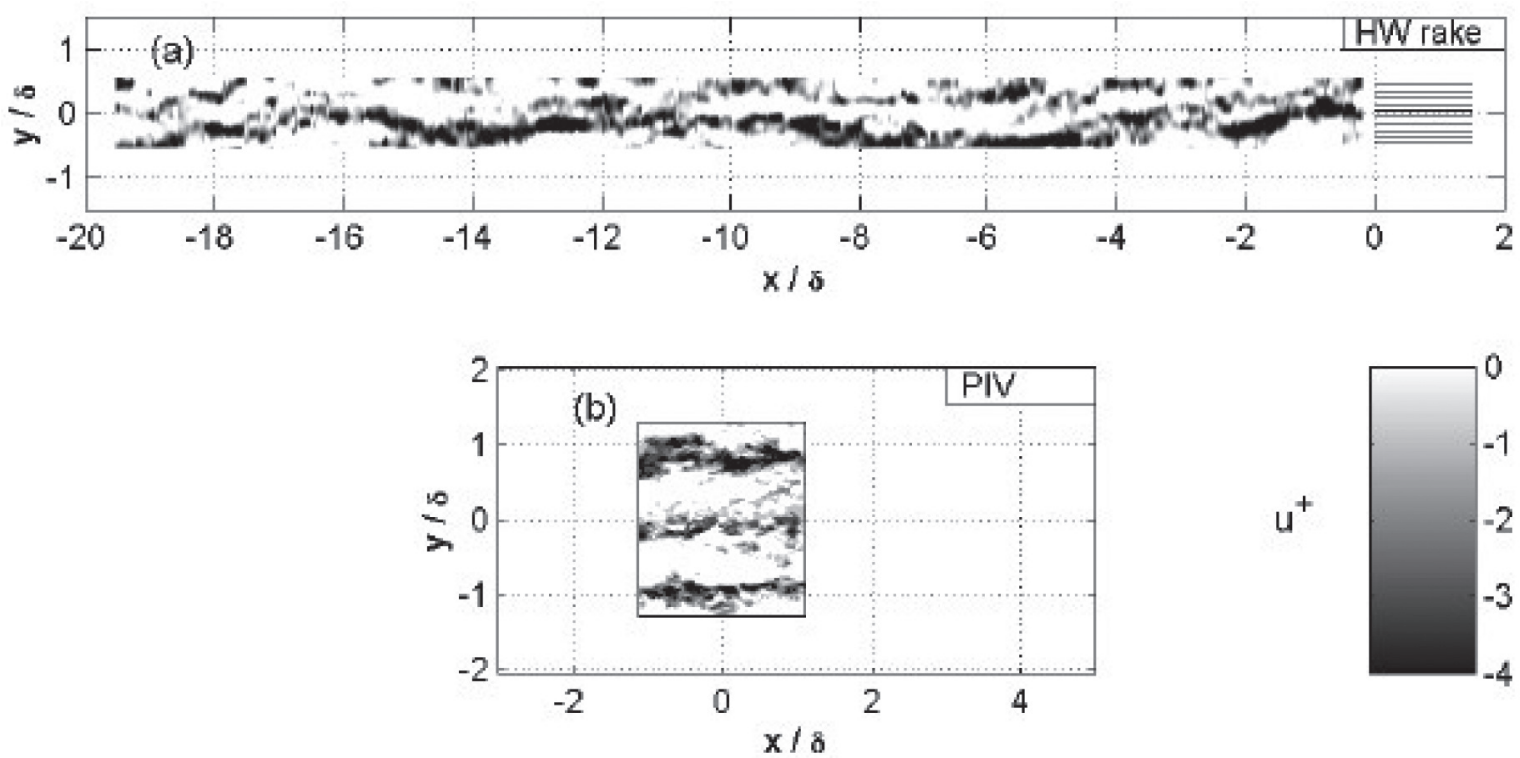

Figure 30 - (a) Example rake signal at $z / \delta=0.15$, for $R e_{\tau}=14380$, and (b) PIV snapshot (from Hutchins \& Marusic 2007a.) Note that $y$ and $z$ are used for spanwise and wall-normal coordinates respectively (the opposite convention to this review). Flow is from left to right. 
As well as being found in the log and lower wake regions of the turbulent boundary layer, these features are also found in pipe and channel flow (Kim and Adrian 1999, Monty et al. 2007). They have been found during PIV experiments (Ganapathisubramani et al. 2006), and in Direct Numerical Simulations (Ringuette et al. 2008). In fact, Balakumar and Adrian (2007) provided the first evidence of VLSMs using energy spectra of pipe, channel and boundary layers quite some time before they were first visualised in any way. They identified two length scales in these wallbounded flows corresponding to peaks in the energy spectra. One peak was observed around $2-3 \delta$ and another around $\approx 6 \delta$. They associated these two length-scales with large-scale motions (LSMs) of $2-3 \delta$ and VLSMs of $\approx 6 \delta$. One can associate these two length scales with the idea of vortex packets, with LSMs being the approximate size of a single vortex packet (see figure 26 for example) and VLSMs being the result of the streamwise concatenation of multiple packets. Dennis and Nickels 2011a provide a picture of such a VLSMs as shown in figure 31. Although the blue region of low momentum appears to be continuous, it is actually two separate regions joined head-over-tail at around $\mathrm{x} / \delta=4.5$, indicating perhaps that two vortex packets have aligned to form this VLSM. By extracting individual LSMs, Dennis and Nickels 2011b went on to demonstrate that it is commonplace for them to align in a streamwise direction and therefore produce the very long regions of low momentum that characterise VLSMs.

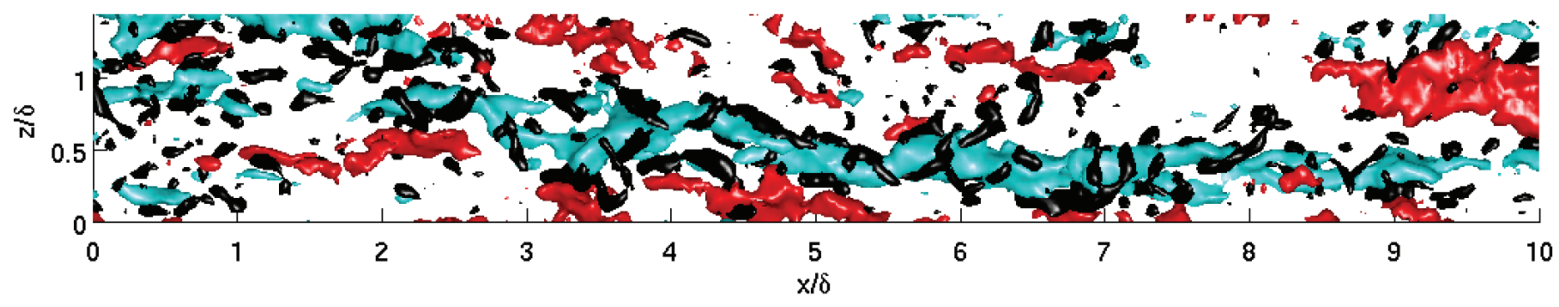

Figure 31 - Top-down view of very large-scale motion draped with vortices (Dennis and Nickels 2011a). Black iso-surface: $\left|\lambda_{\text {ciz }}\right|_{i s o}=0.18\left|\lambda_{\text {ci }}\right|_{\max }$. Blue iso-surface: $u_{i s o}=-0.1 \bar{U}$. Red iso-surface: $u_{i s o}=0.1 \bar{U}$. Flow is from left to right.

One other notable finding from Hutchins and Marusic 2007a was that the one-dimensional pre-multiplied energy spectra of the streamwise velocity fluctuations indicated that the contribution of the VLSMs to these fluctuations increased with increasing Reynolds number. In addition they demonstrated the presence of VLSMs at very high Reynolds numbers $\left(R e_{\tau} \approx 660000\right)$ using an array of sonic anemometers to measure the atmospheric surface layer at the SLTEST site in Utah (Hutchins and Marusic 2007a).

The difference between the different types of wall-bounded turbulent flows has become an area of interest with regard to VLSMs. Monty et al. (2007) used arrays of hot-wires to measure VLSMs in both pipe and channel flow and found that although the VLSMs found in the interior flows were largely similar to those in boundary layers, in the channel flow they extended significantly further from the wall, commensurate with them being a feature of the log-layer and channel flow generally following the log-law further from the wall compared to boundary layers. Lee and Sung (2013) used simulations of both pipe and boundary layer to directly compare the characteristics of the VLSMs inhabiting the two flows. They discovered that the VLSMs in pipe flow were generally much longer (up to $\approx 30 \delta$ ), which they put down to the entrainment occurring in a boundary layer flow causing the more frequent breakdown of the 
streamwise coherence and thus limiting the length of the VLSMs. An aspect of VLSMs that has proved of interest is the proportion of the Reynolds shear stress they contain, which is found to be substantial (Ganapathisubramani et al. 2003, Guala et al. 2006). The results of Lee and Sung (2013) also indicated that the contribution of VLSMs to the Reynolds shear stress was signifcantly higher in a pipe than in a boundary layer.

Baltzer et al. (2013) studied VLSMs in turbulent pipe flow DNS at $R^{+}=685$. Their work reinforced the idea that VLSMs were composed of shorter motions (LSMs) concatenating to form VLSMs. In addition, the two-point spatial correlations showed indications that the VLSMs appear with dominant helix angles (of $\approx 4-5^{\circ}$ ) and "spiral" down the pipe. In fact, there were some hints of this in the hot-wire data of Monty et al. (2007) and it is potentially an interesting feature that is probably peculiar to pipe flow. Baltzer, Adrian and $\mathrm{Wu}$ (2013) also performed proper orthogonal decomposition on the DNS data and the two most energetic modes are shown in figure 32. These display roll-cell motions periodically around the pipe (left-hand-side of figure 32). This pattern is invariant in the streamwise direction except for a rotation about the pipe axis, which gives the POD modes a helical shape (as shown on the righthand-side of figure 32) corresponding to the helical patterns observed in the spatial correlations.

(a)
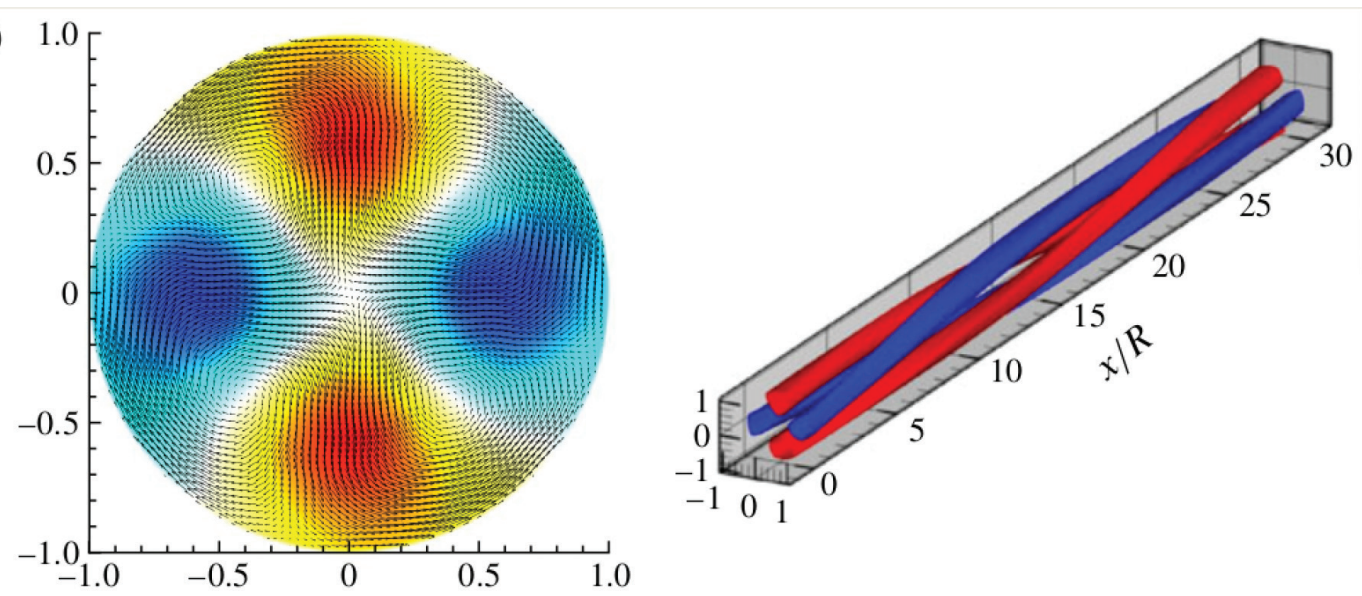

(b)
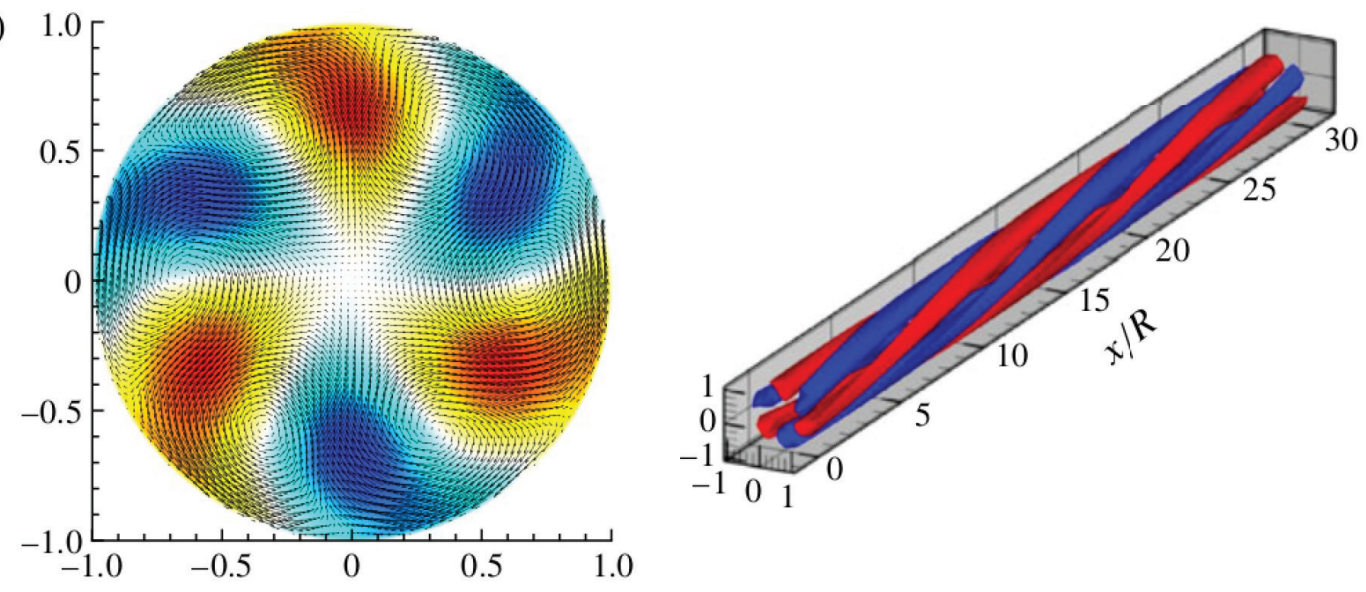

Figure 32 - The two most energetic POD modes displaying roll-cell-like behaviour. The right iso-surfaces indicate the swirling patterns of $u$ (at one-half of maximum magnitude), while the left plots display the colour $u$ fluctuation and in-plane velocity vectors (Baltzer et al. 2013). Flow is in positive $x$ direction. 
Further research on VLSMs has explored their influence on the near-wall motions (Hutchins and Marusic 2007b, Abe et al. 2004). Mathis et al. (2009) reported evidence that the amplitudes of small scale fluctuations near the wall are modulated by the larger scales in the log region. This amplitude modulation was seen to increase with increasing Reynolds number over the range $R e_{\tau} \approx 10^{3}-10^{6}$ (the highest Re being achieved at the atmospheric surface layer at the SLTEST site). Ganapathisubramani et al. (2012) examined the impact on the strength of the large-scale fluctuations on the small-scales and showed that near the wall the small scale amplitude increases with increasing large-scale amplitude, whereas further away from the wall the smallscale amplitude decreases with increasing largescale amplitude. They also found evidence of a frequency modulation effect, but only very close to the wall $\left(\mathrm{y}^{+}<100\right)$ (Ganapathisubramani et al. 2012). A physical mechanism is suggested that reconciles the findings related to this modulation across scales. This involves VLSMs in a hairpin vortex packet-type scenario, the footprint of which, "imposes a large-scale modulation of the nearwall velocity gradient, which in turn modulates the amplitude and frequency of the near-wall cycle"(Ganapathisubramani et al. 2012).

\section{OVERVIEW AND OUTLOOK}

The picture we have of coherent structures in wall-bounded turbulence today is one of families of vortices that arrange themselves in such a way as to create a hierarchy of coherent structures. The base structure is the hairpin-like vortex, which instantaneously does not have to resemble a hairpin, but instead a cane vortex, elongated in the streamwise direction and inclined to the wall. Statistically, however, these vortices are wellrepresented by a hairpin. These vortices tend to appear in packets or clusters that travel together coherently and their effect combines to create a large-scale motion (LSM) which is marked by a region of low momentum fluid (created by the backflow induced by the vortices) approximately $2-3 \delta$ long in the streamwise direction. LSMs themselves tend to concatenate into even longer very large-scale motions (VLSMs), which can be extremely long, $\mathcal{O}(10 \delta)$. The precise mechanism leading to the creation of this arrangement of structures has not been unequivocally defined, although it is clear that they play a very important part in the dynamics of wall-bounded turbulence. In this section some very recent work on the development of new experimental and analytical techniques is discussed in order to provide a view of where wallbounded turbulence research on coherent structures is possibly heading in the near future.

Elsinga et al. (2012) used high-speed tomographic PIV to track the motion of vortical elements in a turbulent boundary layer at $R e_{\theta}=2460$. Tomographic PIV enables the measurement of all three components of velocity in a volume, i.e. truly three-dimensional experimental data. However, due to the difficulties of creating a volume using a laser, the successful tomographic PIV experiments have so far been restricted to measurements using an especially-thick light sheet, rather than a volume covering the whole area of interest. In the case of Elsinga et al. (2012) the measurement volume was $1.8 \delta \times 1.8 \delta$ in streamwise and spanwise direction and $0.11<z / \delta<0.30$ in the wall-normal direction. The field of view is clearly restricted, particularly in the wall-normal direction (in this case). Despite this limitation Elsinga et al. (2012) could track the movement of many individual vortices through this measurement volume and presented statistics on their trajectories. An interesting finding from this study was that the relative velocity between two neighbouring (streamwise-aligned) vortices is small, which is consistent with the idea of vortices travelling coherently together (i.e. in a packet). However they also managed to capture two vortices approaching each other rapidly and merging into a single vortex. 
In a further development of tomo-PIV, Gao et al. (2013) performed moving tomo-PIV in a $\operatorname{Re}_{\theta}=$ 2410 turbulent boundary layer. The measurement system traversed at the local flow velocity and could therefore be used to investigate the evolution of coherent structures in time and space. They were able to observe meandering, merging and breaking of "long slow regions", which are almost certainly parts of VLSMs, although with the restricted fieldof-view $(1650 \times 1650 \times 170$ viscous units (again the shortest dimension is the wall-normal) it is not possible to confirm this unequivocally. They also found that such regions persist in time as they are followed through the boundary layer for distances of $15 \delta$, which corresponds to a time period of $24.3 \delta / U_{\infty}$ or $t^{+}=2300$ (which is just one example). The interaction of VLSMs is inevitable given their scales, but there is more to learn with regard to how it occurs and the effect it has on turbulence production and dissipation. Recently, Lee et al. (2014) investigated the behaviour on LSMs and VLSMs in a DNS of a $\operatorname{Re}_{\tau}=930$ turbulent channel flow using a novel structure detection algorithm. They concluded that VLSMs were primarily formed by the merging of LSMs caused by a relative convection velocity between them. The LSMs themselves were formed through "growing" (lengthening of shorter motions) in addition to merging.

It is clear that time-resolved three-dimensional experimental data could provide a plethora of very interesting insights with regard to coherent structures in wall-turbulence at Reynolds numbers higher than those currently accessible to DNS, but there are still many difficulties in implementing such sophisticated optical techniques in highRe experimental facilities. Overcoming these experimental challenges will be difficult and it is not the only problem that will have to be tackled. The three-dimensional experimental and simulated datasets are so massive that efficient storage and particularly analysis of them is becoming increasingly difficult. Indeed there are almost certainly many secrets hiding in currently collected data that could be uncovered by a full and detailed analysis. One of the aims of future research in wallturbulence should be the development of efficient and inventive analysis techniques that will provide new insights into the phenomenon of turbulence, rather than solely focussing on obtaining more and more experimental and simulated data at increasingly high Reynolds numbers.

VLSMs (or superstructures), being one of the more recently discovered coherent structures, still have a lot of questions surrounding them. Their extreme length is a particularly awkward aspect that is very difficult to precisely define and even more difficult to measure. There existence is also somewhat disconcerting for anybody who believes in the universality (or at least commonality) of turbulent flows. The scaling of VLSMs is an open question, but it is difficult to ignore the possibility that coherent structures with such large streamwise length scales are not entirely independent of the facility in which they are present.

Coherent structures give us a tantalising insight into turbulence and the hope that the daunting chaos of a turbulent flow can be revealed as a combination of relatively simple building-blocks. Despite the great progress described in this review, there is still plenty of work to do. One of the key aspects that needs to be addressed is the link between coherent structures and the governing equations of mechanics fluids (and hence turbulence) the Navier-Stokes equations.

McKeon et al. (2013) and Sharma and McKeon (2013) are rare contributions on coherent structures in wall-bounded turbulence in a NavierStokes framework. Their theory can predict hairpin vortex packets and even VLSM-like structures through a critical layer mechanism associated with the assumed turbulent mean velocity profile. The structures are a result of the superposition of a selected (small) number of response modes (travelling waves), with the addition of different 
modes resulting in increasingly complex structures that bear a remarkable resemblance to those discussed in this review. An example is shown in figure 33. This framework could potentially be a very rich source of insight. In particular it could find applications in the control of turbulent flow through its inherent ability to associate coherent motions with specific response modes and therefore give answers to the critical question of which aspect of a turbulent flow should a control strategy target?
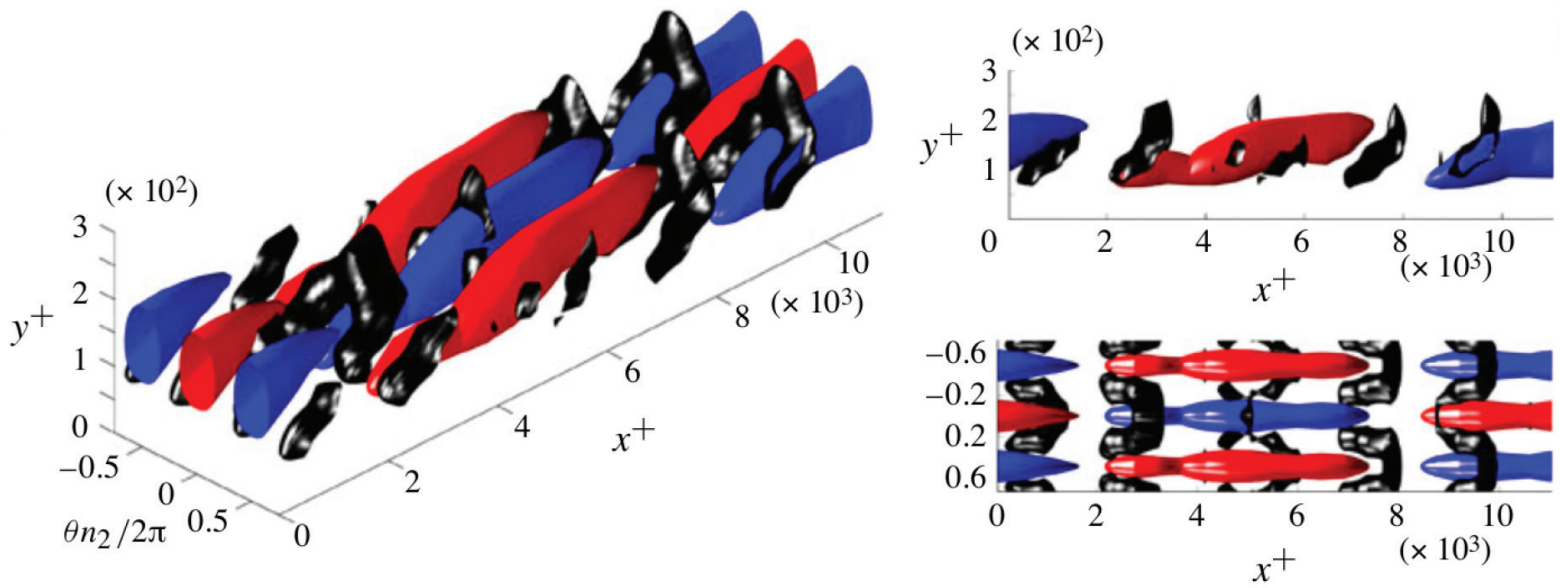

Figure 33 - Model prediction of the swirl field (50\% of maximum) and streamwise velocity ( $\pm 50 \%$ of maximum) arising for a particular mode combination (from Sharma and McKeon 2013). Isosurfaces of swirl are black and isosurfaces of streamwise velocity fluctuation are red and blue for high and low momentum, respectively. Flow is in positive $x$ direction.

Another approach in applying the NavierStokes equations to the issue of coherent structures is a dynamical systems approach to the problem; i.e. to try to capture the essence of turbulence in a mechanistic, low-dimensional system. Importantly, this approach has so far only been effective at very low Reynolds numbers close to transition, but significant advances in turbulence research have been made through applying ideas from finitedimensional dynamical systems theory to the Navier-Stokes equations (Kahawara et al. 2012). This has led to the discovery of "exact coherent states" (Waleffe 1998, 2001), which are essentially invariant solutions of the Navier-Stokes equations, in turbulent flow. A prime example being that of unstable, non-linear travelling wave solutions in a turbulent pipe flow close to transition (Faisst and Eckhardt 2003, Wedin and Keswell 2004, Pringle and Kerswell 2007, Willis and Kerswell 2008, De Lozar et al. 2012, Avila et al. 2013).
The relevance of the "exact coherent states" of dynamical systems to the concepts of "coherent structures" as described in this review is something that it still up for debate. Currently, the biggest divide is the Reynolds number. Whereas experimentalists are often primarily concerned with investigating turbulent behaviour at increasingly high Re, dynamical systems theory is necessarily applied to low-Re flows. This difficulty can lead one to question the relevance of the low-Re exact coherent states unless they can be identified in high-Re turbulent flows, which are evidently more complex than any hitherto identified invariant solution. Despite only being applied at low Re, the dynamical systems viewpoint justifies its relevance to higher Re flow on the basis that there is nothing particularly special about Reynolds numbers near transition and the same mechanistic picture can be applied at any Reynolds number, except that as the Reynolds number increases the number of exact 
solutions also increases. Recent experiments (Dennis and Sogaro 2014) in a large-scale pipe flow facility have provided evidence that flow states reminiscent of recognised travelling-wave solutions are present even at $R e=35000$. This Reynolds number is well above transition and indicates that the relevance of "exact coherent states" may extend to higher Re than previously thought. Importantly this work was also able to show that these states have a statistical imprint on the flow, demonstrating the potential relevance of this approach and providing a fresh perspective on the concept of coherent structures.

\section{RESUMO}

A dificuldade intrínseca à compreensão da turbulência em fluidos estimulou o uso de diferentes metodologias para o tratamento deste problema. Algumas abordagens mostraram-se mais promissoras que outras, mas todas padecem por focar somente em parte do amplo espectro do problema. Uma forma bem sucedida de modelar a turbulência consiste em decompor a estrutura caótica e complexa do escoamento turbulento em um conjunto de movimentos que são, de certa forma, organizados. A esses movimentos atribuímos o termo "estruturas coerentes". Existem várias vertentes desta abordagem, desde a identificação de estruturas coerentes no escoamento, a definição de suas características, a explicação sobre a criação, manutenção e destruição destas estruturas, até a utilização do seu comportamento para a modelagem de escoamentos turbulentos. Este artigo de revisão considera a pesquisa em estruturas coerentes em escoamentos turbulentos de parede: uma classe de escoamento extremamente interessante para muitos cientistas, em particular, mas não exclusivamente, físicos, matemáticos e engenheiros, devido à sua ubiquidade na natureza, na indústria e na vida cotidiana. Esta área da ciência experimentou grande efervescência em anos recentes e parte do seu crescimento foi impulsionado por avanços observados em técnicas experimentais e computacionais. Entretanto, muitas ideias desenvolvidas no passado a partir de intuição e simples visualização do escoamento são ainda hoje muito informativas e relevantes. De fato, a pesquisa atual em estruturas coerentes é reconhecidamente grata aos pioneiros deste tipo de abordagem. Portanto, esta revisão discute aspectos históricos desta linha de pesquisa, bem como aborda os avanços contemporâneos com o intuito de fornecer ao leitor uma boa visão geral do desenvolvimento desta área do conhecimento, destacando a perspicácia de alguns dos primeiros pesquisadores em turbulência. Em suma, este manuscrito fornece um retrato amplo de nossa compreensão atual dos efeitos provocados pelas estruturas coerentes em escoamentos turbulentos de parede.

Palavras-chave: turbulência, escoamentos de parede, estruturas coerentes, velocimetria por imagem de partículas.

\section{REFERENCES}

ABE H, KaWAmura H AND CHOI H. 2004. Very large-scale structures and their effects on the wall shear-stress fluctuations in a turbulent channel flow up to $\mathrm{R} e_{T}=640$. J Fluids Eng 126: 835-843.

ADRIAN RJ. 2007. Hairpin vortex organization in wall turbulence. Phys Fluids 19(4): 041301-041316.

ADRIAN RJ AND LIU ZC. 2002. Observation of vortex packets in direct numerical simulation of fully turbulent channel flow. J Vision 5: 9-19.

Adrian RJ, MeInhart CD AND TOMKIns CD. 2000. Vortex organisation in the outer region of the turbulent boundary layer. J Fluid Mech 422: 1-54.

Avila M, Mellibovsky F, Roland N And Hof B. 2013. Streamwise-localized solutions at the onset of turbulence in pipe flow. Phys Rev Lett 110: 224502.

BALAKUMAR BJ AND ADRIAN RJ. 2007. Large- and very-largescale motions in channel and boundary-layer flows. Phil Trans R Soc A 365(1852): 665-681.

BALTZER JR, ADRIAN RJ AND XIAOHUA Wu. 2013. Structural organization of large and very large scales in turbulent pipe flow simulation. J Fluid Mech 720: 236-279.

BERNARD PS AND WALLACE JM. 2002. Turbulent flow: analysis, measurement and prediction. J Wiley \& Sons, Inc.

BLACK TJ. 1968. An analytical study of the measured wall pressure field under supersonic boundary turbulent boundary layers. NASA CR-888.

CANTwell BJ. 1981. Organised motion in turbulent flow. Ann Rev Fluid Mech 13: 457-515.

Chakraborty P, BALACHANDAR S AND AdRIAN RJ. 2005. On the relationships between local vortex identification schemes. J Fluid Mech 535: 189-214.

Chong MS, Perry AE AND CANTwell BJ. 1990. A general classification of threedimensional flow fields. Phys Fluids A 2(5): 765-777. 
CHRISTENSEN KT AND AdRIAN RJ. 2001. Statistical evidence of hairpin vortex packets in wall turbulence. J Fluid Mech 431: 433-443.

Clark JA AND Markland E. 1971. Flow visualisation in turbulent boundary layers. J Hydraul Div ASCE HY 19: 1653-1664.

CORINO ER AND BRODKEY RS. 1969. A visual investigation of the wall region in turbulent flow. J Fluid Mech 37: 1-30.

Del Alamo JC, Jimenez J, Zandonade P And Moser RD. 2006. Self-similar vortex clusters in the turbulent logarithmic region. J Fluid Mech 561: 329-358.

De Lozar A, Mellibovsky F, Avila M and Hof B. 2012. Edge state in pipe flow experiments. Phys Rev Lett 108: 214502.

DENNIS DJC AND NiCKELS TB. 2008. On the limitations of Taylor's hypothesis in constructing long structures in a turbulent boundary layer. J Fluid Mech 614: 197-206.

DENNIS DJC AND NICKELS TB. 2011a. Experimental measurement of large-scale threedimensional structures in a turbulent boundary layer. Part 1 . Vortex packets. J Fluid Mech 673: 180-217.

DENNIS DJC AND NICKELS TB. 2011b. Experimental measurement of large-scale threedimensional structures in a turbulent boundary layer. Part 2. Long structures. J Fluid Mech 673: 218-244.

DENNIS DJC AND SoGARo FM. 2014. Distinct organizational states of fully developed turbulent pipe flow. Phys Rev Lett 113: 234501.

ELSINGA GE, ADRIAN RJ, VAN OUDHEUSEN BW AND SCARANO F. 2010. Three-dimensional vortex organization in a highReynolds-number supersonic turbulent boundary layer. J Fluid Mech 644: 35-60.

Elsinga GE, PoElma C, Schröder A, GeISLER R, SCARANO F AND WESTERWEEL J. 2012. Tracking of vortices in a turbulent boundary layer. J Fluid Mech 697: 273-295.

FAISST H AND ECKHARDT B. 2003. Traveling waves in pipe flow. Phys Rev Lett 91: 224502.

Ganapathisubramani B, Clemens NT AND Dolling DS. 2006. Large-scale motions in a supersonic turbulent boundary layer. J Fluid Mech 556: 271-282.

GANAPATHISUBRAMANI B, LONGMIRE EK AND MARUSIC I. 2003. Characteristics of vortex packets in turbulent boundary layers. J Fluid Mech 478: 35-46.

Ganapathisubramani B, Hutchins N, Monty JP, Chung D AND MARUSIC I. 2012. Amplitude and frequency modulation in wall turbulence. J Fluid Mech 712: 1-31.

GaO Q, ORTIZ-DueÑas C AND LONGMIRE E. 2013. Evolution of coherent structures in turbulent boundary layers based on moving tomographic PIV. Exp Fluids 54(12): 1-16.

GRASS AJ. 1971. Structural features of turbulent flow over smooth and rough boundaries. J Fluid Mech 50: 233-255.

GuAla M, Hommema SE AND AdRIAN RJ. 2006. Large-scale and very-large-scale motions in turbulent pipe flow. J Fluid Mech 554: 521-542.

GUPTA AK, LAUfER J AND KAPLAN RE. 1971. Spatial structure in the viscous sublayer. J Fluid Mech 50: 493-512.
HAIDARI AH AND SMITH CR. 1994. The generation and regeneration of single hairpin vortices. J Fluid Mech 277: 135-162.

HAMBleton WT, Hutchins N AND Marusic I. 2006. Simultaneous orthogonal-plane particle image velocimetry measurements in a turbulent boundary layer. J Fluid Mech 560: 53-64.

HEAD MR AND BANDYOPADHYAY P. 1981. New aspects of turbulent boundary-layer structure. J Fluid Mech 107: 297-338.

HiNZE JO. 1975. Turbulence. McGraw-Hill, Inc., $2^{\text {nd }}$ ed.

Hommema SE AND AdRIAN RJ. 2003. Packet structure of surface eddies in the atmospheric boundary layer. Boundary-Layer Meteorology 106(1): 147-170.

Hunt JCR, Wray AA AND MoIn P. 1988. Eddies, streams, and convergence zones in turbulent flows. Center for Turbulence Research Report CTR-S88: 193-208.

HuTCHINS N AND MARUSIC I. 2007a. Large-scale inuences in near-wall turbulence. Phil Trans R Soc A 365: 647664.

HutChINS N AND MARUSIC I. 2007b. Evidence of very long meandering features in the logarithmic region of the turbulent boundary layers. J Fluid Mech 579: 1-28.

JEONG J AND HUSSAIN F. 1995. On the identification of a vortex. J Fluid Mech 285: 69-94.

Jimenez J, Hoyas S, Simens MP AND MizunO Y. 2010. Turbulent boundary layers and channels at moderate Reynolds numbers. J Fluid Mech 657: 335-360.

Kawahara G, Uhlmann M And Van Veen L. 2012. The significance of simple invariant solutions in turbulent flows. Ann Rev Fluid Mech 44(1): 203-225.

KASAGI N, HiRATA M AND NisHinO K. 1986. Streamwise pseudovortical structures and associated vorticity in the near-wall region of a turbulent boundary layer. Exp Fluids 4: 309-318.

KIM J AND MoIN P. 1986. The structure of the vorticity field in turbulent channel flow. Part 2. Study of ensembleaveraged fields. J Fluid Mech 162: 339-363.

KIM KC AND ADRIAN RJ. 1999. Very large-scale motion in the outer layer. Phys Fluids A 11(2): 417-422.

KLine SJ, REYNOLdS WC, Schraub FA AND RunSTAdLER PW. 1967. The structure of turbulent boundary layers. J Fluid Mech 30: 741-773.

LEE J, LEE JH, CHOI JI AND SUNG HJ. 2014. Spatial organization of large-and very large-scale motions in a turbulent channel flow. J Fluid Mech 749: 818-840.

LEE JH AND SuNG HJ. 2013. Comparison of very-large-scale motions of turbulent pipe and boundary layer simulations. Phys Fluids 25(4): 045103.

LIU ZC, LANDRETH CC, ADRIAN RJ AND HANRATTY TJ. 1991. High resolution measurement of turbulent structure in a channel with particle image velocimetry. Exp Fluids 10(6): 301-312.

MARUSIC I. 2001. On the role of large-scale structures in wall turbulence. Phys Fluids 13(3): 735-743.

MARUSIC I AND PERRY AE. 1995. A wall-wake model for the turbulence structure of boundary layers. Part 2. Further experimental support. J Fluid Mech 298: 389-407. 
MAThiEU J AND Scott J. 2000. An introduction to turbulent flow. Cambridge University Press.

Mathis R, Hutchins N AND Marusic I. 2009. Large-scale amplitude modulation of the small-scale structures in turbulent boundary layers. J Fluid Mech 628: 311-337.

McKeOn BJ, Sharma AS AND JACOBI I. 2013. Experimental manipulation of wall turbulence: A systems approach. Phys Fluids 25(3): 031301.

MEINHART CD AND ADRIAN RJ. 1995. On the existence of uniform momentum zones in a turbulent boundary layer. Phys Fluids 7(4): 694-696.

MiYAKe Y, USHIRO R AND MORIKAWA T. 1997. The regeneration of quasi-streamwise vortices in the near-wall region. JSME Int J Ser B Fluids Therma Eng 40: 257-264.

MoIN P AND KIM J. 1982. Numerical investigation of turbulent channel flow. J Fluid Mech 118: 341-377.

MoIN P AND KIM J. 1985. The structure of the vorticity field in turbulent channel flow. Part 1. Analysis of instantaneous fields and statistical correlations. J Fluid Mech 155: 441-464.

MONTy JP, SteWART JA, WiLliams RC AND CHONG MS. 2007. Large-scale features in turbulent pipe and channel flows. J Fluid Mech 589: 147-156.

PerRy AE AND CHONG MS. 1982. On the mechanism of wall turbulence. J Fluid Mech 119: 173-217.

Perry AE, Henbest S and Chong MS. 1986. Theoretical and experimental study of wall turbulence. J Fluid Mech 165: 163-199.

Perry AE AND MARUSIC I. 1995. A wall-wake model for the turbulence structure of boundary layers. Part 1. Extension of the attached eddy hypothesis. J Fluid Mech 298: 361-388.

PRINGLE CCT AND KERSWELl RR. 2007. Asymmetric, helical, and mirror-symmetric traveling waves in pipe flow. Phys Rev Lett 99: 074502.

Ringuette MJ, Wu M And PinO MARTin M. 2008. Coherent structures in direct numerical simulation of turbulent boundary layers at Mach 3. J Fluid Mech 594: 59-69.

RobINSON SK. 1991. Coherent motions in the turbulent boundary layer. Ann Rev Fluid Mech 23: 601-639.

SHARMA AS AND MCKEON BJ. 2013. On coherent structure in wall turbulence. J Fluid Mech 728: 196-238.
SMITH CR. 1984. A synthesized model of the near-wall behavior in turbulent boundary layers. In Proc. $8^{\text {th }}$ Symp. Turbulence. University of Missouri-Rolla.

SMITH CR AND METZLER SP. 1983. The mechanism of streak formation in near-wall turbulence. J Fluid Mech 129: 27-54.

SMITH CRAND SCHWARTZ SP. 1983. Observation of streamwise rotation in the near-wall region of a turbulent boundary layer. Phys Fluids 26: 641-652.

TAYLOR GI. 1938. The spectrum of turbulence. In Proc. Roy Soc Lond. A 164: 476-490.

THEODORSEN T. 1952. Mechanism of turbulence. In Proc. $2^{\text {nd }}$ Midwestern Conf. on Fluid Mech, Ohio State University, p. 1-18.

TownSEND AA. 1976. The structure of turbulent shear flow. Cambridge University Press, $2^{\text {nd }}$ ed.

WALEFFE F. 1998. Three-dimensional coherent states in plane shear flows. Phys Rev Lett 81: 4140-4143.

WALEFFE F. 2001. Exact coherent structures in channel flow. J Fluid Mech 435: 93-102.

WEDIN W AND KERSWELL RR. 2004. Exact coherent structures in pipe flow: travelling wave solutions. J Fluid Mech 508: 333-371.

WILLIS AP AND KERSWELL RR. 2008. Coherent structures in localized and global pipe turbulence. Phys Rev Lett 100: 124501.

WILlMARTH WW AND TU BJ. 1967. Structure of turbulence in the boundary layer near the wall. Phys Fluids 10: S137-S137.

WU X AND MoIN P. 2009. Direct numerical simulation of turbulence in a nominally zero-pressure-gradient flat-plate boundary layer. J Fluid Mech 630: 5-41.

ZHOU J, ADRIAN RJ AND BALACHANDAR S. 1996. Autogeneration of near-wall vertical structures in channel flow. Phys Fluids 8(1): 288-290.

ZHOU J, AdRIAN RJ, BALACHANDAR S AND KENDALL TM. 1999. Mechanisms for generating coherent packets of hairpins in channel flow. J Fluid Mech 387: 353-396. 
